\title{
Non-Abelian T-duality as a transformation in Double Field Theory
}

\author{
Aybike Çatal-Özer \\ Department of Mathematics, Istanbul Technical University, \\ Maslak 34469, Istanbul, Turkey \\ E-mail: ozerayb@itu.edu.tr
}

ABSTRACT: Non-Abelian T-duality (NATD) is a solution generating transformation for supergravity backgrounds with non-Abelian isometries. We show that NATD can be described as a coordinate dependent $\mathrm{O}(\mathrm{d}, \mathrm{d})$ transformation, where the dependence on the coordinates is determined by the structure constants of the Lie algebra associated with the isometry group. Besides making calculations significantly easier, this approach gives a natural embedding of NATD in Double Field Theory (DFT), a framework which provides an $\mathrm{O}(\mathrm{d}, \mathrm{d})$ covariant formulation for effective string actions. As a result of this embedding, it becomes easy to prove that the NATD transformed backgrounds solve supergravity equations, when the isometry algebra is unimodular. If the isometry algebra is non-unimodular, the generalized dilaton field is forced to have a linear dependence on the dual coordinates, which implies that the resulting background solves generalized supergravity equations.

Keywords: String Duality, Flux compactifications

ARXIV EPRINT: 1904.00362 


\section{Contents}

1 Introduction 1

2 The action of $O(d, d)$ on curved string backgrounds 4

2.1 Transformation of the fields in the NS-NS sector 4

2.2 Transformation of the fields in the RR sector 6

3 NATD as an $O(d, d)$ transformation $\quad 7$

$\begin{array}{lll}3.1 & \text { An example: } \mathrm{AdS}_{3} \times S^{3} \times T^{4} & 10\end{array}$

4 NATD as a solution generating transformation in Double Field Theory 12

4.1 A brief review of Double Field Theory 12

$\begin{array}{ll}4.2 & \text { Embedding NATD in Double Field Theory } \\ & 14\end{array}$

4.3 Gauged Double Field Theory and fluxes associated with the NATD matrix 17

$\begin{array}{lll}\text { 4.3.1 Gauged Double Field Theory } & 17\end{array}$

4.3.2 Fluxes, dual fluxes and the $O(d, d)$ invariance of GDFT 18

4.3.3 Fluxes associated with the NATD matrix 20

4.4 Comparing the field equations of DFT and GDFT 22

4.4.1 Field equations for the generalized dilaton field 22

$\begin{array}{lll}\text { 4.4.2 } & \text { Field equations for the spinor field } \chi & 23\end{array}$

$\begin{array}{lll}\text { 4.4.3 Field equations for the generalized metric } \mathcal{H}_{M N} & 24\end{array}$

4.5 NATD fields as a solution of DFT in the supergravity frame 26

4.6 Non-unimodular case: generalized supergravity equations 29

5 Conclusions and outlook $\quad 30$

$\begin{array}{ll}\text { A Index conventions } & 31\end{array}$

\section{Introduction}

Non-Abelian T-duality (NATD) is a generalization of T-duality for strings on backgrounds with non-Abelian isometries [1]-[5]. Although it is not as well established as T-duality is as a string duality symmetry, it works well as a solution generating transformation for supergravity. The rules for the transformation of the fields in the NS-NS sector, namely the metric, the B-field and the dilaton field has been known for a long time. Recently, NATD has gained a new interest, as the rules for the transformation of the fields in the RR sector of Type II strings has also been found [6]. This has been applied to many supergravity backgrounds by various groups, especially to backgrounds that are relevant for AdS-CFT correspondence, see for example [7]-[16]. 
Recently, a compact formula for the transformation of the supergravity fields for a generic Green-Schwarz string with isometry $G$ has been obtained in [17], where they also showed that the sigma model after NATD has kappa symmetry. This means that the resulting background is a solution of the generalized supergravity equations (GSE), which have recently been introduced in [18] as a generalization of supergravity equations, see also [19]. To be more precise, when the isometry group $G$ is unimodular, the dualized sigma model is Weyl invariant and the target space is a solution of standard Type II supergravity equations. If $G$ is non-unimodular so that the structure constants of the Lie algebra of $G$ is not traceless, the trace components give rise to a deformation of the equations to be satisfied by the target space fields to GSE. ${ }^{1}$

The purpose of this paper is to describe the NATD transformation rules obtained in [17] as a coordinate dependent $O(10,10)$ transformation. ${ }^{2}$ In Abelian T-duality with $d$ commuting isometries, the transformation rules for the supergravity fields in the NS-NS sector can be neatly described through the action of a constant $O(d, d)$ matrix embedded in $O(10,10)$ [23]. The RR fields are then packaged in a differential form, which can be a regarded as a spinor field that transforms under $\operatorname{Spin}(d, d)$. If the fields in the NS-NS sector transform under $T \in O(d, d)$, then the spinor field that encodes the RR fields transform under $S_{T} \in \operatorname{Spin}(d, d)$, which is the element that projects onto $T$ under the double covering homomorphism $\rho$ between $O(d, d)$ and $\operatorname{Spin}(d, d)$, that is, $\rho(S)=T$ [24]. In a similar fashion, we show in this paper that the NATD transformation of the supergravity fields in the NS-NS sector can be described through the action of an $O(10,10)$ matrix (presented in (3.20)), this time not constant but with an explicit dependence on the coordinates of the dual theory. The dependence on the coordinates is determined by the structure constants of the Lie algebra associated with the isometry group. The transformation of the RR fields is then automatically determined by the corresponding $\operatorname{Spin}(d, d)$ matrix, as in Abelian T-duality. We would like to note that we had already presented the NATD matrix we give in this paper at a workshop at APCTP, Pohang [27]. Very recently, a paper has appeared which also views NATD as an $O(d, d)$ transformation [25]. See also [26], which has a similar approach to NATD.

Besides making calculations significantly easier, our approach makes it possible to view NATD as a solution generating transformation in Double Field Theory (DFT), a framework which provides an $O(d, d)$ covariant formulation for effective string actions [28][38] by introducing dual, winding type coordinates. In its current formulation, DFT is a consistent field theory only when a certain constraint, called the strong constraint is satisfied. If the DFT fields have no dependence on the winding type coordinates, the strong constraint is satisfied trivially and the fields are said to be in the supergravity frame. In such a case, DFT of Type II strings constructed in [34, 38] reduces to Type II supergravity

\footnotetext{
${ }^{1}$ The fact that the NATD background fails to satisfy standard supergravity equations when $G$ is nonunimodular was first noted in [20] and the generalized equations appeared first in [21]. For a detailed account, see [22].

${ }^{2}$ The $O(10,10)$ matrix associated with the NATD transformation is obtained by embedding an $O(d, d)$ matrix in $O(10,10)$, where $d$ is the dimension of the isometry group. Hence, the only non-trivial action is on the isometry directions. For this reason, we will sometimes refer to this action as an $O(d, d)$ transformation.
} 
in the democratic formulation. When the duality group is unimodular, the NATD fields always belong to the supergravity frame, and hence our method provides a simple proof of the fact that the transformed fields solve supergravity equations, when the isometry algebra is unimodular. If the isometry algebra is non-unimodular, we show that the generalized dilaton field of DFT is forced to have a linear dependence on the winding type coordinates. In such a coordinate frame, DFT equations are known to reduce to generalized supergravity equations $[39,40]$. This then implies that the resulting NATD fields should solve GSE, consistent with what has been found in the literature so far. Let us make a remark at this point. The NATD rules for the NS-NS sector and the fact that it is a solution generating transformation for supergravity has been known since early 90's, as has been mentioned before. These rules were obtained directly from the sigma model by applying the Buscher procedure. On the other hand, the rules for the fields in the RR sector has been figured out only recently in [6], by "guessing" them from how the Buscher rules extend to the RR sector in Abelian T-duality (and initially only for the Principal Chiral Model). This approach does not provide a proof of why the transformed fields should constitute a proper supergravity background, so it had to be checked example by example that the NATD fields indeed solved (generalized) supergravity equations. Embedding NATD in DFT as we do here provides a proof that this should always be the case. Our approach in this paper is quite different from that of [17], where they also prove that the dualized fields satisfy the GSE by checking the kappa symmetry of the transformed Green-Schwarz sigma model. Here, we consider the transformation of the fields within DFT and directly check that the dual fields satisfy the field equations of DFT in an appropriate frame, where they reduce to (generalized) supergravity equations. NATD has been studied in the context of DFT also in the papers $[41,42]$. There is a generalized notion of T-duality, called the Poisson Lie T-Duality [43, 44], which does not require the symmetry group $G$ to act by isometries. It includes Abelian T-duality and NATD as special cases. Poisson Lie T-duality has been studied in the context of DFT in $[45]^{3}$ and very recently in [25].

As the $O(10,10)$ matrix that produces the NATD fields is not constant, it is not immediately clear that it generates a solution generating transformation for DFT. To show that this is indeed the case, we find it useful to utilize the framework of Gauged Double Field Theory (GDFT), which is obtained by a duality twisted (Scherk-Schwarz) reduction [49, 50] of DFT [51]-[54]. GDFT is a deformation of DFT, determined by the fluxes associated with the twist matrix that define the duality twisted reduction anzats. Our strategy is as follows: we start with a solution of Type II supergravity. Since Type II supergravity can be embedded in DFT, one can construct corresponding DFT fields which constitute a solution for DFT in the supergravity frame. If the space-time metric has an isometry symmetry $G$, which is also respected by the B-field and the RR fluxes (not necessarily the gauge potentials), we can extract DFT fields out of the original ones, which satisfy the field equations of GDFT determined by the geometric flux associated with the isometry group $G$. We call these fields untwisted DFT fields. The DFT fields

\footnotetext{
${ }^{3}$ To be more precise, [45] studies Poisson Lie T-duality in the framework of DFT on group manifolds (usually called DFTwzw), which is a different theory from the standard DFT. DFT on group manifolds was constructed in the papers [46-48].
} 
corresponding to the NATD background are obtained by acting on these untwisted fields with the $O(10,10)$ NATD matrix we present here. We will show that these dual fields also satisfy the field equations of DFT by using the following three key facts, which we will prove in the body of the paper: i) Field equations of GDFT are $O(d, d)$ (or $\operatorname{Spin}(d, d)$ ) covariant, provided we also allow fluxes transform as generalized tensors. ii) By using fact (i) above, we show that a set of duality twisted DFT fields, which we generically write as $\phi(x, Y)=U(Y) \cdot \phi(x),{ }^{4}$ satisfy the field equations of DFT if and only if the untwisted fields $\phi(x)$ satisfy the field equations of GDFT determined by the fluxes associated by the twist matrix $U$. This immediately implies the following: a set of fields $\tilde{\phi}(x, Z)=\tilde{U}(Z) \cdot \phi(x)$, where the twist matrix $\tilde{U}(Z)$ produces the same fluxes as $U(Y)$, will satisfy the field equations of DFT if and only if the fields $\phi(x, Y)=U(Y) . \phi(x)$ satisfy the field equations DFT. iii) The fluxes associated with the isometry group $G$ and the NATD matrix $T$ are exactly the same. Facts ii) and iii) together prove that the NATD fields indeed form a solution of DFT, as claimed.

The structure of the paper is as follows: in the next section, we give a brief review of the $O(d, d)$ structure of Abelian T-duality, first in the NS-NS sector and then in RR sector in subsection 2.2. This enables us to identify the coordinate dependent $O(d, d)$ matrix that generates the NATD background in section 3. Also in this section (in subsection 3.1), we demonstrate how the NATD of the background $\mathrm{AdS}_{3} \times S_{3} \times T^{4}$ studied in [6] can be obtained by the action of the NATD matrix we have identified. Then in section 4 , we study the embedding of NATD in DFT. The three key facts that we listed in the previous paragraph are proved in this section. The distinction between the unimodular and nonunimodular cases is also discussed here. We finish the paper with discussions and outlook in section 5 .

Note added. While we were about to finalize the writing of this manuscript, the paper [25] appeared on the arXiv, parts of which overlap with the work we present here.

\section{The action of $O(d, d)$ on curved string backgrounds}

In this section, we review how Abelian T-duality can be described as an $O(d, d)$ transformation, first for the NS-NS sector and then for the RR sector. For the RR sector, the duality group should be lifted to $\operatorname{Spin}(d, d)$. We closely follow [23] in section 2.1 and [24] in section 2.2 .

\subsection{Transformation of the fields in the NS-NS sector}

Let $g$ and $B$ be the metric and the Kalb-Ramond 2-form field that describes a $D$ dimensional supergravity background, with d commuting isometries. The string living on this background exhibits an $O(d, d, Z)$ T-duality symmetry. Accordingly, there is an $O(d, d, R)$ action, which acts as a solution generating symmetry in the low energy limit. Let us define

\footnotetext{
${ }^{4}$ Here, the action of $U(Y) \in O(d, d)$ is determined by how $\phi$ transforms under $O(d, d)$ or more generally under $\operatorname{Spin}(d, d)$ if $\phi$ is a spinor field in DFT.
} 
the $D \times D$ background matrix

$$
Q(G, B)=G+B
$$

Due to the presence of $\mathrm{d}$ commuting isometries, it is possible to choose adopted coordinates $X=\left(x^{I}, x^{m}\right), I=1, \cdots, d$ such that the background matrix does not depend on the $d$ coordinates $x^{I}$. Let us decompose the background matrix with respect to this choice of coordinates as

$$
Q=\left(\begin{array}{ll}
Q_{I J} & Q_{I m} \\
Q_{m I} & Q_{m n}
\end{array}\right)=\left(\begin{array}{cc}
E & F^{2} \\
F^{1} & F
\end{array}\right) .
$$

Let $T$ be a matrix in $O(d, d, R)$. Then

$$
T=\left(\begin{array}{ll}
a & b \\
c & d
\end{array}\right), \quad a^{t} c+c^{t} a=0, \quad b^{t} d+d^{t} b=0, \quad a^{t} d+c^{t} b=I .
$$

This can be embedded in $O(D, D, R)$ as follows

$$
\hat{T}=\left(\begin{array}{ll}
\hat{a} & \hat{b} \\
\hat{c} & \hat{d}
\end{array}\right)
$$

where $\hat{a}, \hat{b}, \hat{c}, \hat{d}$ are $D \times D$ matrices defined below:

$$
\hat{a}=\left(\begin{array}{ll}
a & 0 \\
0 & I
\end{array}\right), \hat{b}=\left(\begin{array}{ll}
b & 0 \\
0 & 0
\end{array}\right), \hat{c}=\left(\begin{array}{ll}
c & 0 \\
0 & 0
\end{array}\right), \hat{d}=\left(\begin{array}{ll}
d & 0 \\
0 & I
\end{array}\right) .
$$

Let

$$
Q^{\prime}\left(G^{\prime}, B^{\prime}\right)=\hat{T} \cdot Q(G, B) \equiv(\hat{a} Q+\hat{b})(\hat{c} Q+\hat{d})^{-1}
$$

be the new background matrix obtained by the above action of $O(D, D, R)$ on $Q$. Then, it is well known that the transformed metric and the transformed B-field obtained from

$$
G^{\prime}=\frac{Q^{\prime}+Q^{\prime t}}{2}, \quad B^{\prime}=\frac{Q^{\prime}-Q^{\prime t}}{2}
$$

define (along with the transformed dilaton field we will discuss below, see (2.10)) valid supergravity backgrounds. That is, the $O(D, D, R)$ transformation defined above acts as a solution generating transformation.

For completeness, let us write the final form of the transformed background matrix $Q^{\prime}$ :

$$
Q^{\prime}=\left(\begin{array}{cc}
E^{\prime} & \left(a-E^{\prime} c\right) F^{2} \\
F^{1}(c E+d)^{-1} & F-F^{1}(c E+d)^{-1} c F^{2}
\end{array}\right)
$$

where

$$
E^{\prime}=T \cdot E=(a E+b)(c E+d)^{-1} .
$$

For the resulting background to be a valid supergravity solution, the dilaton field $\phi$ should also transform under $O(D, D, R)$ in the following way:

$$
e^{-2 \phi^{\prime}}=e^{-2 \phi} \sqrt{\frac{\operatorname{det} G}{\operatorname{det} G^{\prime}}}
$$


For later reference, we define the following field $d$, which is invariant under $O(D, D)$ transformations:

$$
d=\phi-\frac{1}{4} \ln \operatorname{det} G
$$

Note that this gives

$$
e^{-2 d}=\sqrt{\operatorname{det} G} e^{-2 \phi}
$$

It is easily checked from (2.10) that $e^{-2 d^{\prime}}=e^{-2 d}$ under $O(D, D)$. The field $d$ will play an important role in DFT as the generalized dilaton field, as we will discuss in section 4.1.

\subsection{Transformation of the fields in the RR sector}

Let us now discuss how the p-form fields in the RR sector of Type II supergravity theory transform under the action of $O(d, d, R)$ described in the section above. For this discussion we closely follow [24], see also [55].

In the democratic formulation of Type II supergravity [56], the 0,2 and 4-form fields in Type IIB and 1 and 3 form fields in Type IIA are combined with their Hodge duals to form sections of the exterior bundle $\bigwedge^{\text {even }} T^{*} M$ for the first case and of $\bigwedge^{\text {odd }} T^{*} M$ for the latter, where $M$ is the space-time manifold. It is well known that these bundles carry the chiral spinor representations of $\operatorname{Pin}(d, d)$, which is the double covering group of $O(d, d)$. The transformation of the RR fields under T-duality is determined by this action of $\operatorname{Pin}(d, d)$ on the RR fields, viewed as a section of the exterior bundle. More precisely, if the T-duality transformation in the NS-NS sector is realized by the $O(d, d)$ matrix $T$, then the $\operatorname{Pin}(d, d)$ element acting on the spinor field that packages the modified RR gauge potentials is $S$, where $\rho(S)=T \cdot{ }^{5}$ Here, $\rho$ is the double covering map

$$
\rho: \operatorname{Pin}(d, d) \rightarrow O(d, d)
$$

Then, if $\chi$ is the spinor field that packages the modified RR fields we have

$$
\chi \rightarrow \chi^{\prime}=S \chi
$$

Let us now discuss the transformation of the field strength $\not \partial \chi$ under $\operatorname{Pin}(d, d)$. This is important since RR fluxes are defined as

$$
F=e^{-B \not \partial \chi}
$$

As was discussed in [24], the transformation (2.14) does not imply $\not \partial \chi \rightarrow S \not \partial \chi$. However, when one doubles the space-time coordinates as in Double Field Theory (DFT), which we will discuss in more detail in section $4.1, \not \partial \chi$ also transforms as a vector under $\operatorname{Pin}(d, d)$ as $\not \partial \chi \rightarrow S \not \partial \chi$. In DFT, the usual space-time coordinates are doubled by introducing winding type coordinates, which combine with the space-time coordinates to form an $O(d, d)$ vector $X^{M}=\left(\tilde{x}_{\mu}, x^{\mu}\right)$ that transforms as $X^{M} \rightarrow X^{M}=T_{N}^{M} X^{N}$ (see section 4.1). This implies

\footnotetext{
${ }^{5}$ See the papers [38] and [54] for an overview of $O(d, d)$ and its double covering group Pin $(d, d)$. The method to find the $\operatorname{Pin}(d, d)$ matrix corresponding to a given $O(d, d)$ matrix is also explained in these papers.
} 
$\partial_{M} \rightarrow \partial_{M}^{\prime}=\left(T^{-1}\right)_{M}^{N} \partial_{N}$. Now, using $S^{-1} \Gamma^{M} S=T_{P}^{M} \Gamma^{P}$, which follows directly from $\rho(S)=T$, one can show

$$
\begin{aligned}
\not \partial \chi & =\Gamma^{M} \partial_{M} \chi \rightarrow \not \partial^{\prime} \chi^{\prime}=\Gamma^{M}\left(T^{-1}\right)^{N}{ }_{M} \partial_{N}(S \chi) \\
& =S S^{-1} \Gamma^{M} S\left(T^{-1}\right)^{N}{ }_{M} \partial_{N} \chi=S \not \partial \chi .
\end{aligned}
$$

The transformation of $\not \partial \chi$ implies that

$$
F=e^{-B} \not \partial \chi \rightarrow F^{\prime}=e^{-B^{\prime}} \not \not^{\prime} \chi^{\prime}=e^{-B^{\prime}} S \not \partial \chi=e^{-B^{\prime}} S e^{B} F
$$

\section{NATD as an $O(d, d)$ transformation}

Non-Abelian T-duality can be applied by using the standard tools of the Buscher method. For a generic nonlinear sigma model with isometry group $G$, one starts with gauging the symmetry group (or a subgroup of it) and introduces Lagrange multiplier terms which constrains the gauge field to be pure gauge. Integrating out the Lagrange multipliers, one obtains the original model. Integrating out the gauge field gives the NATD model, for which the Lagrange multiplier terms play the role of coordinates on the dual manifold.

The NATD of a generic Green-Schwarz string sigma model with isometry group $G$ has been recently obtained in [17]. Here we present their results (for bosonic $G$ ) and show that the new backgrounds can also be obtained by applying a coordinate dependent $O(d, d)$ transformation embedded in $O(10,10)$. The best way to present the rules for transformation is to introduce coordinates which makes the isometry symmetry manifest. With respect to such coordinates one can write

$$
\begin{aligned}
d s^{2} & =G_{\mu \nu} d x^{\mu} d x^{\nu}=G_{m n} d x^{m} d x^{n}+2 G_{m i} d x^{m} d \theta^{i}+G_{i j} d \theta^{i} d \theta^{j} \\
& =G_{m n} d x^{m} d x^{n}+2 G_{m I} d x^{m} \sigma^{I}+G_{I J} \sigma^{I} \sigma^{J} \\
& =G_{\alpha \beta} \sigma^{\alpha} \sigma^{\beta}
\end{aligned}
$$

where $\theta^{i}, i=1, \cdots, d$ are coordinates for $G$ and $\sigma^{a}=\delta_{m}^{a} d x^{m}$ and $\sigma^{I}, I=1, \cdots, \operatorname{dim} G$ are the left invariant 1-forms $\sigma^{I}=l^{I}{ }_{i} d \theta^{i}$ on $G$ defined from the Maurer-Cartan form: $g^{-1} d g=\sigma^{I} T_{I}$ with $T_{I}$ forming a basis for the Lie algebra $\mathcal{G}$ of $G$. Similarly,

$$
\begin{aligned}
B & =\frac{1}{2} B_{\mu \nu} d x^{\mu} \wedge d x^{\nu} \\
& =\frac{1}{2} B_{m n} d x^{m} \wedge d x^{n}+B_{m I} d x^{m} \wedge \sigma^{I}+\frac{1}{2} B_{I J} \sigma^{I} \wedge \sigma^{J}
\end{aligned}
$$

Since the group $G$ acts on the background by isometries, all the $\theta$ dependence of the fields are encoded in $l_{i}^{I}$. After applying NATD with respect to $G$, one ends up with a sigma 
model which corresponds to the following background ${ }^{6}$

$$
\begin{aligned}
G_{m n}^{\prime} & =G_{m n}-[(G+B) N(G+B)]_{(m n)} \\
G_{m I}^{\prime} & =\frac{1}{2}[(G+B) N]_{m I}-\frac{1}{2}[N(G+B)]_{I m} \\
G_{I J}^{\prime} & =N_{(I J)} \\
B_{m n}^{\prime} & =B_{m n}-[(G+B) N(G+B)]_{[m n]} \\
B_{m I}^{\prime} & =-\frac{1}{2}[(G+B) N]_{m I}-\frac{1}{2}[N(G+B)]_{I m} \\
B_{I J}^{\prime} & =-N_{[I J]},
\end{aligned}
$$

where

$$
N^{I J}=\left(G_{I J}+B_{I J}+\nu_{K} C_{I J}^{K}\right)^{-1} .
$$

Here, $C_{I J}{ }^{K}$ are the structure constants of the Lie algebra $\mathcal{G}$ with respect to the basis $T_{I}$, that is, $\left[T_{I}, T_{J}\right]=C_{I J}{ }^{K} T_{K}$. The metric and the B-field in the transformed background are

$$
\begin{aligned}
d s^{2} & =G_{m n}^{\prime} d x^{m} d x^{n}+2 G_{m I}^{\prime} d x^{m} d \nu^{I}+G_{I J}^{\prime} d \nu^{I} d \nu^{J} \\
B^{\prime} & =\frac{1}{2} B_{m n}^{\prime} d x^{m} \wedge d x^{n}+B_{m I}^{\prime} d x^{m} \wedge d \nu^{I}+\frac{1}{2} B_{I J}^{\prime} d \nu^{I} \wedge d \nu_{J} .
\end{aligned}
$$

Here, $\nu_{I}$ are Lagrange multiplier terms in the Buscher method. They parameterize the dual space and hence they have lower indices as in (3.12). Those indices are raised by the Kronecker delta $\nu^{I}=\delta^{I J} \nu_{J}$ in (3.13), (3.14) so that they have the standard upper placement of indices as coordinates of the NATD fields. This has also been discussed in [17], see their footnote 7 .

The transformation for the dilaton field presented in [17] is

$$
\phi^{\prime}=\phi+\frac{1}{2} \ln \operatorname{det} N \text {. }
$$

Now, let us write the above transformation rules in the terminology of the previous section. We define the background matrix $Q=G+B$ and $Q^{\prime}=G^{\prime}+B^{\prime}$. Then the above rules become

$$
\begin{aligned}
Q_{m n}^{\prime} & =Q_{m n}-\left[\left(Q_{m I} N^{I J} Q_{J n}\right]\right. \\
Q_{m I}^{\prime} & =[Q N]_{m I} \\
Q_{I m}^{\prime} & =-[N Q]_{I m} \\
Q_{I J}^{\prime} & =N_{I J}
\end{aligned}
$$

Comparing this with (2.8) and (2.9) one immediately sees that the new background has been obtained by the action of the fractional linear transformation with the following $O(d, d)$ matrix $T_{\text {NATD }}$ embedded in $O(10,10),{ }^{7}$ in the way presented in the section above:

$$
T_{\mathrm{NATD}}=\left(\begin{array}{cc}
0 & 1 \\
1 & \theta_{I J}
\end{array}\right), \quad \theta_{I J}=\nu_{K} C_{I J}^{K} .
$$

\footnotetext{
${ }^{6}$ Note that due to the convention in [17] there is a difference in the sign in front of the B field term.

${ }^{7}$ We name both matrices (the $O(d, d)$ matrix $(3.20)$ and the $O(10,10)$ matrix in which it is embedded) as $T_{\mathrm{NATD}}$.
} 
Let us also check that the transformation rule (3.15) for the dilaton field can be obtained through the action of $T_{\text {NATD }}$ by comparing it with (2.10). It is a well known fact that the transformation (2.6) implies for $G^{\prime}$ the following [23]:

$$
G^{\prime}=\frac{1}{(\hat{c} Q+\hat{d})^{T}} G \frac{1}{(\hat{c} Q+\hat{d})} .
$$

Then,

$$
\frac{\operatorname{det} G^{\prime}}{\operatorname{det} G}=\left(\operatorname{det}(\hat{c} Q+\hat{d})^{-1}\right)^{2} .
$$

When $T$ is as in (3.20) this gives

$$
\sqrt{\frac{\operatorname{det} G^{\prime}}{\operatorname{det} G}}=\operatorname{det} N
$$

and the two expressions (2.10) and (3.15) indeed match.

It is important to note that the dimension $d$ of the isometry group determines whether the NATD matrix $T_{\text {NATD }}$ acts within Type IIA/Type IIB or it involves a reflection which implies that a Type IIA solution is mapped to a Type IIB solution or vice versa. The former situation arises when $d$ is even and the latter occurs when $d$ is odd.

Since we have identified the $O(10,10)$ matrix that generates the NS-NS sector of the NATD background, we can immediately determine the transformed RR sector, as well. All we have to do is to find the Pin $(10,10)$ matrix that acts on the spinor field that packages the modified p-form gauge potentials in the democratic formulation. The Pin $(10,10)$ element $S_{\text {NATD }}$ that projects to the $O(10,10)$ element $(3.20)$ under the double covering homomorphism $\rho: \operatorname{Pin}(d, d) \rightarrow O(d, d)$ can be found easily:

$$
S_{\mathrm{NATD}}=C S_{\theta}=S_{\beta} C,
$$

where $C$ is the charge conjugation matrix. For more details, see [38] and [54]. The factors $S_{\theta}$ and $S_{\beta}$ in $S_{\text {NATD }}$ are the $\operatorname{Spin}^{+}(10,10)$ elements that projects onto the $\mathrm{SO}^{+}(10,10)$ matrix that generates the $B$-transformations and $\beta$-shifts with $\theta_{I J}=\nu_{K} C_{I J}^{K}$ and $\beta_{I J}=\nu_{K} C_{I J}{ }^{K}$, respectively. Then the transformation of the p-form fluxes is

$$
F^{\prime}=e^{-B^{\prime}} S_{\mathrm{NATD}} e^{B} F .
$$

An important remark is in order here. Recall from the discussion in section 2.2 that the transformation (2.17) is equivalent to the transformation (2.16). Also recall that the transformation (2.16) equivalent to the transformation (2.14), when $S$ is constant. However, when $S$ is not constant as in here, the two transformations (2.16) and (2.14) are not equivalent. Naively, one would have expected that the right transformation rule for the RR fields under NATD would follow from the transformation

$$
\chi \rightarrow S_{\text {NATD }} \chi,
$$

which would imply a different transformation rule for the field strength $F$ that would also involve fluxes associated with $S_{\text {NATD }}$ (see section 4.3.2). However, the right transformation rule is as in (3.25), as we will demonstrate in the next section through the example of the $\mathrm{AdS}_{3} \times S^{3} \times T^{4}$. Then in section 4.4.2, we will prove that the transformed fields will constitute a solution of the GSE when the transformation for the RR fields is as in (3.25). 


\subsection{An example: $\mathrm{AdS}_{3} \times S^{3} \times T^{4}$}

Let us consider the simple example $\mathrm{AdS}_{3} \times S^{3} \times T^{4}$. This geometry arises as the near horizon limit of the D1-D5 system. The geometry has to be supported by 3 -form Ramond-Ramond flux. We have

$$
\begin{aligned}
d s^{2} & =d s^{2}\left(\operatorname{AdS}_{3}\right)+d s^{2}\left(S^{3}\right)+d s^{3}\left(T^{4}\right) \\
F_{3} & =\operatorname{Vol}\left(S^{3}\right)+\operatorname{Vol}\left(\mathrm{AdS}_{3}\right)
\end{aligned}
$$

Note that we also need the Hodge dual of the 3 -form flux which is the following 7 -form flux:

$$
F_{7}=-\left(\star F_{3}\right)=\left(\operatorname{Vol}\left(S^{3}\right)+\operatorname{Vol}\left(\operatorname{AdS}_{3}\right)\right) \wedge \operatorname{Vol}\left(T^{4}\right) .
$$

Due to the presences of the 3 -sphere in the geometry, one has a global $\mathrm{SO}(4) \simeq$ $\mathrm{SU}(2) \times \mathrm{SU}(2)$ isometry symmetry. It is possible to use one of these $\mathrm{SU}(2)$ groups to apply NATD. Writing the $S^{3}$ part of the metric as

$$
d s^{2}\left(S^{3}\right)=\left(\sigma^{1}\right)^{2}+\left(\sigma^{2}\right)^{2}+\left(\sigma^{2}\right)^{2},
$$

where $\sigma^{I}, I=1,2,3$ are the 3 left invariant 1 -forms for $\mathrm{SU}(2)$, we have

$$
Q_{m I}=Q_{I m}=0
$$

and

$$
Q_{I J}=E_{I J}=G_{I J}=\delta_{I J} .
$$

Now we apply the NATD matrix (3.20) on this background. Here, the structure constants that determine the NATD matrix are $C_{I J}^{K}=\epsilon_{I J}^{K}$. This gives

$$
\begin{aligned}
d s^{2}\left(S_{\mathrm{def}}^{3}\right) & =\frac{1}{1+r^{2}} \sum_{I, J=1}^{3}\left(\left(1+\nu^{I}\right)^{2}\left(d \nu^{I}\right)^{2}+2 \nu^{I} \nu^{J} d \nu^{I} d \nu^{J}\right) \\
B^{\prime} & =\frac{1}{1+r^{2}}\left(-\nu^{3} d \nu^{1} \wedge d \nu^{2}+\nu^{2} d \nu^{1} \wedge d \nu^{3}-\nu^{1} d \nu^{2} \wedge d \nu^{3}\right),
\end{aligned}
$$

where $r^{2}=\left(\nu^{1}\right)^{2}+\left(\nu^{2}\right)^{2}+\left(\nu^{3}\right)^{2}$. Writing this in spherical coordinates

$$
\nu^{1}=r \sin \theta \cos \phi \quad \nu^{2}=r \sin \theta \sin \phi \quad \nu^{3}=r \cos \theta
$$

we have

$$
\begin{aligned}
d s^{2} & =d s^{2}\left(\operatorname{AdS}_{3}\right)+d s^{2}\left(\tilde{S}^{3}\right)+d s^{3}\left(T^{4}\right) \\
d s^{2}\left(\tilde{S}^{3}\right) & =d r^{2}+\frac{r^{2}}{1+r^{2}} d \Omega^{2}=d r^{2}+\frac{r^{2}}{1+r^{2}}\left(d \theta^{2}+\sin ^{2} \theta d \phi^{2}\right) \\
B^{\prime} & =-\frac{r^{3}}{1+r^{2}} \operatorname{Vol}\left(S^{2}\right)=-\frac{r^{3}}{1+r^{2}} \sin \theta d \theta \wedge d \phi \\
\Phi^{\prime} & =-\frac{1}{2} \ln \left(1+r^{2}\right)
\end{aligned}
$$


Now, let us look at the transformation of the RR sector. Similar to the Abelian case we form the differential form, which encodes the RR fluxes

$$
F=\sum_{p} G^{(p)}=\sum_{p}\left(F^{(p)}+F_{I}^{(p-1)} \sigma^{I}+\frac{1}{2} F_{I J}^{(p-2)} \sigma^{I} \wedge \sigma^{J}+F^{(p-3)} \sigma^{1} \wedge \sigma^{2} \wedge \sigma^{3}\right)
$$

where we have decomposed a $p$-form $\mathrm{RR}$ flux $G^{(p)}$ according to how many legs it does have along the directions of the isometry group $\mathrm{SU}(2)$. The fluxes $F^{(p-a)}, a=0,1,2,3$ have no dependence on the coordinates $r, \theta, \phi$. We map this differential form to a Clifford algebra element in the usual way. The difference we have here is that it is $\sigma^{I}$ and not $d x^{i}$ that we identify with the Clifford algebra element $\psi^{I}$, for $I=1,2,3$. On the other hand, for $a=d+1, \cdots, 10, d x^{a}$ is replaced with $\psi^{a}$, as usual. Here, $\psi^{\alpha}, \alpha=(I, a)$ are the Clifford algebra elements $\psi^{\alpha}=1 / \sqrt{2} \Gamma^{\alpha}$, where $\Gamma^{\alpha}$ are the Gamma matrices. For more details, see [54]. For index conventions, see appendix A. For the example we consider in this section we only have 3 - and 7-form fluxes, so the spinor field takes the following form:

$$
\begin{aligned}
F= & \psi^{1} \cdot \psi^{2} \cdot \psi^{3}+F^{(3)} \psi^{\hat{1}} \cdot \psi^{\hat{2}} \cdot \psi^{\hat{3}} \\
& +F^{(3)} F^{(4)} \psi^{\hat{1}} \cdot \psi^{\hat{2}} \cdot \psi^{\hat{3}} \psi^{\hat{4}} \cdot \psi^{\hat{5}} \cdot \psi^{\hat{6}} \psi^{\hat{7}}+F^{(4)} \psi^{1} \cdot \psi^{2} \cdot \psi^{3} \psi^{\hat{4}} \cdot \psi^{\hat{5}} \cdot \psi^{\hat{6}} \psi^{\hat{7}} .
\end{aligned}
$$

Here, $\operatorname{Vol}\left(\mathrm{AdS}_{3}\right)=F^{(3)} d x^{\hat{1}} \wedge d x^{\hat{2}} \wedge d x^{\hat{3}}$ and $\operatorname{Vol}\left(T^{4}\right)=F^{(4)} d x^{\hat{4}} \wedge d x^{\hat{5}} \wedge d x^{\hat{6}} \wedge d x^{\hat{7}}$. Here, the hatted numbers count the non-isometric directions. Note that $F^{(3)}$ and $F^{(4)}$ are functions, not forms. Now we calculate $F^{\prime}$ from (3.25). First note that $e^{B} F=F$, since the B-field is zero on the original background. Let us first calculate $S_{\theta} F=\left(1+\nu_{K} \epsilon_{I J}^{K} \psi^{I} \cdot \psi^{J}\right)$.F. As one can easily calculate, this gives

$$
\begin{aligned}
S_{\theta} \cdot F & =F+\nu_{K} \epsilon_{I J}^{K} \psi^{I} \cdot \psi^{J} \cdot F \\
& =F+F^{(3)} \nu_{K} \epsilon_{I J}^{K} \psi^{I} \cdot \psi^{J} \cdot \psi^{\hat{1}} \cdot \psi^{\hat{2}} \cdot \psi^{\hat{3}}\left(1+F^{(4)} \psi^{\hat{4}} \cdot \psi^{\hat{5}} \cdot \psi^{\hat{6}} \psi^{\hat{7}}\right) .
\end{aligned}
$$

Now we apply the charge conjugation operator [38]

$$
C \equiv\left(\psi^{1}-\psi_{1}\right) \cdot\left(\psi^{2}-\psi_{2}\right) \cdot\left(\psi^{3}-\psi_{3}\right)
$$

on (3.41):

$$
\begin{aligned}
C S_{\theta} \cdot F= & C \cdot F-F^{(3)} \nu_{K} \psi^{K} \psi^{\hat{1}} \cdot \psi^{\hat{2}} \cdot \psi^{\hat{3}}\left(1+F^{(4)} \psi^{\hat{4}} \cdot \psi^{\hat{5}} \cdot \psi^{\hat{6}} \psi^{\hat{7}}\right) \\
= & 1+F^{(4)} \psi^{\hat{4}} \cdot \psi^{\hat{5}} \cdot \psi^{\hat{6}} \psi^{\hat{7}}+F^{(3)} \psi^{1} \cdot \psi^{2} \cdot \psi^{3} \cdot \psi^{\hat{1}} \cdot \psi^{\hat{2}} \cdot \psi^{\hat{3}}\left(1+F^{(4)} \psi^{\hat{4}} \cdot \psi^{\hat{5}} \cdot \psi^{\hat{6}} \psi^{\hat{7}}\right) \\
& -F^{(3)} \nu_{K} \psi^{K} \psi^{\hat{1}} \cdot \psi^{\hat{2}} \cdot \psi^{\hat{3}}\left(1+F^{(4)} \psi^{\hat{4}} \cdot \psi^{\hat{5}} \cdot \psi^{\hat{6}} \psi^{\hat{7}}\right) \cdot
\end{aligned}
$$

Finally, we apply $e^{-B^{\prime}}=1+\frac{1}{1+r^{2}} \nu_{K} \epsilon_{I J}^{K} \psi^{I} \cdot \psi^{J}$ on (3.43), where we read off $B^{\prime}$ from (3.34):

$$
\begin{aligned}
F^{\prime}=e^{-B^{\prime}} C S_{\theta} F= & 1+F^{(4)} \psi^{\hat{4}} \cdot \psi^{\hat{5}} \cdot \psi^{\hat{6}} \psi^{\hat{7}} \\
& +\left(1-\frac{r^{2}}{1+r^{2}}\right) F^{(3)} \psi^{1} \cdot \psi^{2} \cdot \psi^{3} \cdot \psi^{\hat{1}} \cdot \psi^{\hat{2}} \cdot \psi^{\hat{3}}\left(1+F^{(4)} \psi^{\hat{4}} \cdot \psi^{\hat{5}} \cdot \psi^{\hat{6}} \psi^{\hat{7}}\right) \\
& -\nu_{K} \psi^{K} F^{(3)} \psi^{\hat{1}} \cdot \psi^{\hat{2}} \cdot \psi^{\hat{3}}\left(1+F^{(4)} \psi^{\hat{4}} \cdot \psi^{\hat{5}} \cdot \psi^{\hat{6}} \psi^{\hat{7}}\right) \\
& +\frac{1}{1+r^{2}} \nu_{K} \epsilon_{I J}{ }^{K} \psi^{I} \cdot \psi^{J} \cdot\left(1+F^{(4)} \psi^{\hat{4}} \cdot \psi^{\hat{5}} \cdot \psi^{\hat{6}} \psi^{\hat{7}}\right)
\end{aligned}
$$


From $F^{\prime}$ we can read off the p-form fluxes of the dual background after now identifying $\psi^{I}$ with $d \nu^{I}$. Since it is only the $S^{3}$ directions that have been dualized, we still have

$$
F^{(4)} \psi^{\hat{4}} \cdot \psi^{\hat{5}} \cdot \psi^{\hat{6}} \psi^{\hat{7}} \leftrightarrow \operatorname{Vol}\left(T^{4}\right) \text { and } F^{(3)} \psi^{\hat{1}} \cdot \psi^{\hat{2}} \cdot \psi^{\hat{3}} \leftrightarrow \operatorname{Vol}\left(\mathrm{AdS}_{3}\right),
$$

The fluxes in the NATD background are then found as

$$
\begin{aligned}
F_{0} & =1 \\
F_{2} & =\frac{1}{1+r^{2}}\left(\nu^{1} d \nu^{2} \wedge d \nu^{3}+\nu^{2} d \nu^{3} \wedge d \nu^{1}+\nu^{3} d \nu^{1} \wedge d \nu^{2}\right)=\frac{r^{3}}{1+r^{2}} \operatorname{Vol}\left(S^{2}\right) \\
F_{4} & =\operatorname{Vol}\left(T^{4}\right)-\sum_{I=1}^{3} \nu^{I} d \nu^{I} \operatorname{Vol}\left(\operatorname{AdS}_{3}\right)=\operatorname{Vol}\left(T^{4}\right)-r d r \operatorname{Vol}\left(\mathrm{AdS}_{3}\right) \\
F_{6} & =\frac{1}{1+r^{2}}\left[d \nu^{1} \wedge d \nu^{2} \wedge d \nu^{3} \wedge \operatorname{Vol}\left(\operatorname{AdS}_{3}\right)+\left(\nu^{1} d \nu^{2} \wedge d \nu^{3}+\nu^{2} d \nu^{3} \wedge d \nu^{1}+\nu^{3} d \nu^{1} \wedge d \nu^{2}\right) \operatorname{Vol}\left(T^{4}\right)\right] \\
& =\frac{r^{2}}{1+r^{2}} d r \wedge \operatorname{Vol}\left(S^{2}\right) \wedge \operatorname{Vol}\left(\operatorname{AdS}_{3}\right)+\frac{r^{3}}{1+r^{2}} \operatorname{Vol}\left(S^{2}\right) \wedge \operatorname{Vol}\left(T^{4}\right) \\
& =\operatorname{Vol}\left(\tilde{S}^{3}\right) \wedge \operatorname{Vol}\left(\operatorname{AdS}_{3}\right)+\frac{r^{3}}{1+r^{2}} \operatorname{Vol}\left(S^{2}\right) \wedge \operatorname{Vol}\left(T^{4}\right) \\
F_{8} & =-\sum_{I=1}^{3} \nu^{I} d \nu^{I} \wedge \operatorname{Vol}\left(\operatorname{AdS} S_{3}\right) \wedge \operatorname{Vol}\left(T^{4}\right)=-r d r \wedge \operatorname{Vol}\left(\operatorname{AdS}_{3}\right) \wedge \operatorname{Vol}\left(T^{4}\right) \\
F_{10} & =\operatorname{Vol}\left(\tilde{S}^{3}\right) \wedge \operatorname{Vol}\left(\operatorname{AdS}_{3}\right) \wedge \operatorname{Vol}\left(T^{4}\right)=\star 1
\end{aligned}
$$

Here,

$$
\operatorname{Vol}\left(\tilde{S}^{3}\right)=\frac{r^{2} \sin \theta}{1+r^{2}} d r \wedge d \theta \wedge d \phi=\frac{1}{1+r^{2}} \operatorname{Vol}\left(S^{3}\right)=\frac{r^{2}}{1+r^{2}} d r \operatorname{Vol}\left(S^{2}\right),
$$

and $\star$ is the Hodge dual with respect to the metric of the deformed background given in (3.36). These results match exactly with the results obtained in [6] by conventional methods of NATD.

\section{NATD as a solution generating transformation in Double Field Theory}

The purpose of this section is to show that the NATD fields obtained by applying the transformation (2.6) and (3.25), where $T$ in (2.4) is the NATD matrix (3.20) are solutions of (generalized) supergravity equations. We find it useful to discuss this in the framework of Double Field Theory (DFT), where $O(d, d)$ arises as a manifest symmetry of the action and hence of the field equations. Therefore, we start with a brief review of DFT.

\subsection{A brief review of Double Field Theory}

DFT is a field theory defined on a doubled space, which implements the $O(d, d)$ T-duality symmetry of string theory as a manifest symmetry. In addition to the standard spacetime coordinates, the doubled space also includes dual coordinates, which are associated with the winding excitations of closed string theory on backgrounds with non-trivial cycles. 
The space-time and the dual coordinates transform as a vector under the T-duality group $O(d, d)$ :

$$
X^{M}=h^{M}{ }_{N} X^{N}, \quad X^{M}=\left(\begin{array}{c}
\tilde{x}_{\mu} \\
x^{\mu}
\end{array}\right)
$$

Here $\tilde{x}_{\mu}$ are the dual coordinates and $h^{M}{ }_{N}$ is a general $O(d, d)$ matrix. In what follows we will always decompose the indices $M$ labelling the $O(d, d)$ representation as ${ }^{M}=\left({ }_{\mu},{ }^{\mu}\right)$, where ${ }^{\mu}$ and ${ }_{\mu}$ label representations of the GL $(d)$ subgroup of $O(d, d)$. We will raise and lower indices by the $O(d, d)$ invariant metric $\eta$, so that $X_{M}=\eta_{M N} X^{N}$.

In DFT, the dynamical fields are the fields $\mathcal{H}, \mathbb{S}, d$ and $\chi$. They are all allowed to depend on both the standard and the winding type coordinates. The generalized metric $\mathcal{H}$ is an element of $\mathrm{SO}^{-}(d, d)$ and it encodes the semi-Riemannian metric and the B-field, see (4.17). The generalized dilaton field $d$ is defined from $e^{-2 d}=\sqrt{g} e^{-2 \phi}$ and it is $O(d, d)$ invariant as was discussed in section 2.1, see (2.12). The spinor field $\mathbb{S}$ is the element in $\operatorname{Spin}^{-}(d, d)$ that projects onto $\mathcal{H}$ under the double covering homomorphism (2.13) between $\operatorname{Spin}(d, d)$ and $\mathrm{SO}(d, d)$, that is $\rho(\mathbb{S})=\mathcal{H}$. The spinor field $\chi$ encodes the RR fields in the democratic formulation of Type II supergravity. For more details see [38, 54].

The DFT action is as below:

$$
\mathcal{S}=\int d x d \tilde{x}\left(\mathcal{L}_{\mathrm{NS}-\mathrm{NS}}+\mathcal{L}_{\mathrm{RR}}\right)
$$

where

$$
\mathcal{L}_{\mathrm{NS}-\mathrm{NS}}=e^{-2 d} \mathcal{R}(\mathcal{H}, d)
$$

and

$$
\mathcal{L}_{\mathrm{RR}}=\frac{1}{4}\left\langle\not \partial \chi, C^{-1} \mathbb{S} \not \partial \chi\right\rangle .
$$

Here, \langle\rangle is the Mukai pairing, which is a $\operatorname{Spin}(d, d)$ invariant bilinear form on the space of spinors [57]. This action has to be implemented by the following self-duality condition

$$
\not \partial \chi=-\mathcal{K} \not \partial \chi, \quad \mathcal{K} \equiv C^{-1} \mathbb{S} .
$$

Moreover, one needs to impose the following $O(d, d)$ covariant constraint, which is called the strong constraint:

$$
\partial^{M} \partial_{M} A=\eta^{M N} \partial_{M} \partial_{N} A=0, \quad \partial^{M} A \partial_{M} B=0, \quad \eta^{M N}=\left(\begin{array}{ll}
0 & 1 \\
1 & 0
\end{array}\right)
$$

where $A$ and $B$ represent any fields or parameters of the theory. When the constraint is satisfied, the DFT action is gauge invariant under generalized diffeomorphisms and the gauge algebra closes under the C-bracket, which is an $O(d, d)$ covariant extension of the Courant bracket. When the fields and the gauge parameters have no dependence on the winding type coordinates, that is, when $\tilde{\partial}^{\mu}=0$, the strong constraint (4.6) is satisfied trivially. In this case, the theory is said to be in the supergravity frame because for this solution of the constraint it can be shown that (4.3) reduces to the standard NS-NS action 
for the massless fields of string theory and (4.4) reduces to the RR sector of the democratic formulation of Type II supergravity theory.

The term $\mathcal{R}(\mathcal{H}, d)$ in (4.3) is the generalized Ricci scalar and its explicit form is as follows:

$$
\begin{aligned}
\mathcal{R}(\mathcal{H}, d)= & 4 \mathcal{H}^{M N} \partial_{M} \partial_{N} d-\partial_{M} \partial_{N} \mathcal{H}^{M N}-4 \mathcal{H}^{M N} \partial_{M} d \partial_{N} d+4 \partial_{M} \mathcal{H}^{M N} \partial_{N} d \\
& +\frac{1}{8} \mathcal{H}^{M N} \partial_{M} \mathcal{H}^{K L} \partial_{N} \mathcal{H}_{K L}-\frac{1}{2} \mathcal{H}^{M N} \partial_{M} \mathcal{H}^{K L} \partial_{K} \mathcal{H}_{N L}
\end{aligned}
$$

The DFT action presented in (4.4) is invariant under the following transformations:

$$
\mathbb{S}(X) \longrightarrow \mathbb{S}^{\prime}\left(X^{\prime}\right)=\left(S^{-1}\right)^{\dagger} \mathbb{S}(X) S^{-1}, \quad \chi(X) \longrightarrow \chi\left(X^{\prime}\right)=S \chi(X)
$$

Here $S \in \operatorname{Spin}(d, d)$ and $X^{\prime}=h X$, where $h=\rho(S) \in \mathrm{SO}^{+}(d, d)$. As mentioned before, the generalized dilaton field is $O(d, d)$ invariant. The transformation rules for the generalized metric $\mathcal{H}=\rho(\mathbb{S})$ is determined by those of $\mathbb{S}$ and is as given below:

$$
\mathcal{H}(X) \longrightarrow \mathcal{H}^{\prime}\left(X^{\prime}\right)=\left(h^{-1}\right)^{T} \mathcal{H}(X) h^{-1} .
$$

The generalized Ricci scalar (4.7) is manifestly invariant under these transformations. A fact that is of crucial importance is that the transformation (4.9) is equivalent to [35]

$$
Q \rightarrow Q^{\prime}=(A Q+B)(C Q+D)^{-1},
$$

where

$$
h=\left(\begin{array}{ll}
A & B \\
C & D
\end{array}\right) .
$$

\subsection{Embedding NATD in Double Field Theory}

We showed in the previous section that the NATD of a given Type II background with isometry $G$ can be obtained through the action of the $O(d, d)$ matrix (3.20). As we have mentioned before, the field equations of DFT reduce to the field equations of Type II supergravity for the trivial solution of the constraint, that is when the fields are in the supergravity frame. As a result, the type II supergravity solution on which the NATD acts can also be regarded as a solution for DFT. Now, assume that the isometry group $G$ is unimodular ${ }^{8}$ and that it acts freely on the background. The latter condition means that one can pick up coordinates in which the metric and the B-field can be written as in (3.2) and (3.5). We label these coordinates as $\left\{x^{1}, \cdots, x^{10-d}, \theta^{1}, \cdots, \theta^{d}\right\}$; then the dual coordinates will be labelled as $\left\{\tilde{x}^{1}, \cdots, \tilde{x}^{10-d}, \tilde{\theta}^{1}, \cdots, \tilde{\theta}^{d}\right\}$. Obviously, the DFT fields $\mathcal{H}, \mathbb{S}, d, \chi$ that correspond to this background do not depend on the dual coordinates, that is, they are in the supergravity frame.

Since the group $G$ acts on the background by isometries, all the $\theta$ dependence of the fields in (3.2) and (3.5) are encoded in $l^{I}$. We define the matrices $G(x, \theta), G(x), B(x, \theta)$ and $B(x)$ from

$$
d s^{2}=d \mathbf{x}^{T} G(x, \theta) d \mathbf{x}=\sigma^{T} G(x) \sigma, \quad B=d \mathbf{x}^{T} B(x, \theta) \wedge d \mathbf{x}=\sigma^{T} B(x) \wedge \sigma,
$$

\footnotetext{
${ }^{8}$ We will relax the condition of unimodularity later on.
} 
where $\wedge$ denote the obvious wedge product of matrices and $d \mathbf{x}$ and $\sigma$ denote the 10-vectors with components $\left(d x^{1}, \cdots, d x^{10}\right)$ and $\left(\sigma^{1}, \cdots, \sigma^{d}, d x^{d+1}, \cdots, d x^{10}\right)$, respectively. Then the background matrix $Q=G+B$ in (2.1) has the following form:

$$
Q(x, \theta)=l^{T}(\theta) Q(x) l(\theta),
$$

where $l$ is the GL(10) matrix obtained by embedding the GL( $d)$ matrix $l_{d}$ with components $\left(l_{d}\right)_{i}^{I}=l_{i}^{I}$. The embedding is as described in (2.5), so $\left(l_{d}\right)_{m}^{I}=l_{i}^{a}=0$ and $\left(l_{d}\right)^{a}{ }_{m}=\delta_{m}^{a}$. This is equivalent to the following $O(10,10)$ action:

$$
Q(x, \theta)=L(\theta) \cdot Q(x)
$$

where $L$ is the $O(10,10)$ matrix

$$
L=\left(\begin{array}{cc}
l^{T} & 0 \\
0 & l^{-1}
\end{array}\right) .
$$

As stated at the end of section 4.1, the equation (4.13) is equivalent to [35]:

$$
\mathcal{H}(x, \theta)=L(\theta) \mathcal{H}(x) L^{T}(\theta) .
$$

Hence, the dependence of the generalized metric $\mathcal{H}$ on the coordinates $(x, \theta)$ is separated. Since the twist matrix $L$ operates between curved and flat indices, the index structure of it is as follows:

$$
\mathcal{H}^{M N}\left(x^{1}, \cdots, x^{10-d}, \theta^{1}, \cdots, \theta^{d}\right)=L_{A}^{M}\left(\theta^{1}, \cdots, \theta^{d}\right) \mathcal{H}^{A B}\left(x^{1}, \cdots, x^{10-d}\right) L^{N}{ }_{B}\left(\theta^{1}, \cdots, \theta^{d}\right),
$$

where we have identified

$$
\mathcal{H} \longleftrightarrow \mathcal{H}^{M N} \longleftrightarrow\left(\begin{array}{cc}
G-B G^{-1} B & G^{-1} \\
-G^{-1} B & G^{-1}
\end{array}\right) .
$$

From (4.14) we read off $L_{m}^{a}=\delta_{m}^{a}, L_{a}^{m}=\delta_{a}^{m}, L_{m}^{I}=L_{I}^{m}=L_{i}^{a}=L_{a}^{i}=0$ and $L_{i}{ }^{I}=\left(L_{d}\right)_{i}{ }^{I}=l_{i}{ }^{I}, L^{i}{ }_{I}=\left(L_{d}\right)^{i}{ }_{I}=l^{i}{ }_{I}$, where $l^{i}{ }_{I}{ }^{I}{ }_{j}=\delta^{i}{ }_{j}$.

Similarly, the dependence of the field $\mathbb{S}$ on the coordinates $(x, \theta)$ is also separated.

$$
\mathbb{S}\left(x^{1}, \cdots, x^{10-d}, \theta^{1}, \cdots, \theta^{d}\right)=\left(S_{L}^{-1}\right)^{\dagger}\left(\theta^{1}, \cdots, \theta^{d}\right) \mathbb{S}\left(x^{1}, \cdots, x^{10-d}\right)\left(S_{L}\right)^{-1}\left(\theta^{1}, \cdots, \theta^{d}\right)
$$

Here, $S_{L}$ is the Pin $(10,10)$ matrix that projects onto $L$ under the double covering homomorphism: $\rho\left(S_{L}\right)=L$. For $\mathcal{K} \equiv C^{-1} \mathbb{S}$, this implies

$$
\mathcal{K}(x, \theta)=S_{L}(\theta) \mathcal{K}(x) S_{L}^{-1}(\theta) .
$$

We also assume that the p-form field strengths (not the gauge potentials) respect this isometry, that is, we assume that any p-form flux in the background can be written as

$$
G^{(p)}=\sum_{p}\left(F^{(p)}(x)+F_{I}^{(p-1)}(x) \sigma^{I}+\frac{1}{2} F_{I J}^{(p-2)}(x) \sigma^{I} \wedge \sigma^{J}+\cdots+F^{(p-d)} \sigma^{1} \wedge \cdots \wedge \sigma^{d}\right) .
$$


Here, we have decomposed a $p$-form RR flux $G^{(p)}$ according to how many legs it does have along the directions of the isometry group $G$. Since $G$ acts by isometries, the fluxes $F^{(p-a)}(x), a=0,1, \cdots, d$ will have no dependence on the isometry coordinates $\theta^{i}$. Let $F$ be the differential form that packages these $\mathrm{p}$-forms as in the democratic formulation:

$$
F=\sum_{p} G^{(p)}
$$

This can be regarded as a spinor field as discussed in section 3, for more details, see [38] and [54]. Owing to the form (4.20), we have

$$
F(x, \theta)=S_{L}(\theta) F(x)
$$

where $F(x, \theta)$ is the spinor field that encodes the components of the field strengths written with respect to the coordinate basis $\left(d x^{1}, \cdots, d x^{(10-d)}, d \theta^{1}, \cdots, d \theta^{d}\right)$. As we will show in a moment, when the twist matrix is of the form (4.14), (4.22) is equivalent to the following

$$
F(x, \theta)=e^{-B(x, \theta)} S_{L}(\theta) e^{B(x)} F(x) .
$$

As we have mentioned before, the relation (4.23) above is equivalent to

$$
\not \partial \chi\left(x^{1}, \cdots, x^{n-d}, \theta^{1}, \cdots, \theta^{d}\right)=S_{L}\left(\theta^{1}, \cdots, \theta^{d}\right) \not \partial \chi\left(x^{1}, \cdots, x^{n-d}\right) .
$$

In order to show the equivalence of the equations (4.22) and (4.23), first note that

$$
S_{L}^{-1}(\theta) e^{B(x, \theta)} S_{L}(\theta)=e^{B(x)} .
$$

This follows from (4.11), which implies that $B(x, \theta)=l^{T} B(x) l$. Writing

$$
h_{B}=\left(\begin{array}{ll}
1 & B \\
0 & 1
\end{array}\right) \text {, }
$$

this means

$$
L^{-1} h_{B(x, \theta)} L=h_{B(x)}
$$

Then, we have

$$
\rho\left(S_{L}^{-1}\right) \rho\left(e^{B(x, \theta)}\right) \rho\left(S_{L}\right)=\rho\left(e^{B(x)}\right),
$$

where $\rho$ is the double covering homomorphism $\rho: \operatorname{Spin}(d, d) \rightarrow O(d, d)$. Note that we have used $\rho\left(e^{B}\right)=h_{B}$ and $\rho\left(S_{L}\right)=L$. Now, $\rho$ is a homomorphism so the left hand side can be rewritten as $\rho\left(S_{L}^{-1} e^{B(x, \theta)} S_{L}\right)$. This then gives $(4.25)$, as desired. Using this we immediately get

$$
e^{-B(x, \theta)} S_{L}(\theta) e^{B(x)} F(x)=e^{-B(x, \theta)} S_{L}(\theta) \underbrace{S_{L}^{-1}(\theta) e^{B(x, \theta)} S_{L}(\theta)} F(x)=S_{L}(\theta) F(x),
$$

where the indicated terms is written by using (4.25).

Using the terminology from duality twisted (Scherk-Shwarz) reduction that we will discuss in subsection 4.3.1, we call the fields $\mathcal{H}(x), \mathbb{S}(x), d(x)$ and $F(x)$ untwisted fields. 
Now, we apply the NATD transformation (2.6) and (3.25) on these untwisted fields, where $T$ in (2.4) is as in (3.20). This will give us the dual fields $\mathcal{H}^{\prime}, d^{\prime}, \mathbb{S}^{\prime}$ and $F^{\prime}$, which will depend on the coordinates $\left\{x^{1}, \cdots, x^{10-d}, \nu^{1}, \cdots, \nu^{d}\right\}$, which we collectively call $\{x, \nu\}$.

$$
\begin{aligned}
\mathcal{H}^{\prime M N}(x, \nu) & =\left(T_{\mathrm{NATD}}\right)^{M}{ }_{A}(\nu) \mathcal{H}^{A B}(x)\left(T_{\mathrm{NATD}}\right)^{N}{ }_{B}(\nu) \\
\mathcal{K}(x, \nu) & =S_{\mathrm{NATD}}(\nu) \mathcal{K}(x)\left(S_{\mathrm{NATD}}\right)^{-1}(\nu) \\
F^{\prime}(x, \nu) & =e^{-\sigma(\nu, \tilde{\nu})} e^{-B^{\prime}(x, \nu)} S_{\mathrm{NATD}}(\nu) e^{B(x)} F(x) \\
d^{\prime}(x, \nu) & =d(x)+\sigma(\nu, \tilde{\nu}) .
\end{aligned}
$$

Here, $\rho\left(S_{\text {NATD }}\right)=T_{\text {NATD }}$ and $B^{\prime}(x, \nu)$ is read off from the antisymmetric part of $\mathcal{H}^{\prime}(x, \nu)$ in (4.30). The field $\sigma(\nu, \tilde{\nu})$ in (4.33) and (4.32) is non-vanishing only when the isometry group is non-unimodular. We leave the discussion of this term to section 4.6.

Our strategy will be to show that these new fields $\mathcal{H}^{\prime}(x, \nu), d^{\prime}(x, \nu), \mathbb{S}^{\prime}(x, \nu)$ and $F^{\prime}(x, \nu)$ form a solution for the field equations of DFT. Identifying the coordinates $\{x, \nu\}$ with the standard space-time coordinates, this means that the corresponding supergravity fields in the NATD background form a solution for the field equations of Type II supergravity, as the field equations of DFT and Type II supergravity are equivalent in the supergravity frame.

The key point in our argument will be to show that the two twist matrices $L(\theta)$ and $T_{\mathrm{NATD}}(\nu)$ generate the same fluxes defined in the framework of Gauged Double Field Theory(GDFT). In the next section, we give a brief review of GDFT, and introduce the fluxes that arise in this context. Finally, we compute the fluxes associated with $L$ and $T_{\text {NATD }}$ and show that they are indeed the same.

\subsection{Gauged Double Field Theory and fluxes associated with the NATD matrix}

\subsubsection{Gauged Double Field Theory}

GDFT is obtained from duality twisted (Scherk-Schwarz) reduction of DFT [51]-[54]. The $O(d, d)$ invariance of the DFT action under the transformations (4.8) and (4.9) makes it possible to introduce the following Scherk-Schwarz type reduction anzats for the DFT fields:

$$
\begin{aligned}
\mathcal{H}^{M N}(x, Y) & =\left(U^{-1}\right)^{M}{ }_{A}(Y) \mathcal{H}^{A B}(x)\left(U^{-1}\right)^{N}{ }_{B}(Y), \quad \mathcal{K}(x, \theta)=S(Y) \mathcal{K}(x) S^{-1}(Y) \\
F(x, Y) & =e^{-\sigma(Y)} e^{-B(x, Y)} S(Y) e^{B(x)} F(x), \\
d(x, Y) & =d(x)+\sigma(Y),
\end{aligned}
$$

where $\rho(S)=U^{-1} \in O(d, d)$ and $F(x, Y)=e^{-B(x, Y) \not \partial \chi}(x, Y)$. The matrices $U$ and $S$ are usually called twist matrices. When these anzatse are plugged into the action and the gauge transformation rules of DFT, all the $Y$ dependence is integrated out and one ends up with GDFT, which is a consistent field theory for the untwisted fields $\mathcal{H}(x), \mathcal{K}(x), d(x), F(x)$, provided that the matrices $U(Y), S(Y)$ satisfy a set of constraints. We will list these constraints in section 4.3.2. The GDFT action is a deformation of the DFT action determined by the so called fluxes, $f_{A B C}$. In the NS-NS sector the Ricci scalar in (4.3) is deformed to

$$
\mathcal{R} \rightarrow \mathcal{R}_{\mathrm{def}}=\mathcal{R}+\mathcal{R}_{f},
$$


with

$$
\begin{aligned}
\mathcal{R}_{f}= & -\frac{1}{2} f_{B C}^{A} \mathcal{H}^{B D} \mathcal{H}^{C E} \partial_{D} \mathcal{H}_{A E}-\frac{1}{12} f_{B C}^{A} f_{E F}^{D} \mathcal{H}_{A D} \mathcal{H}^{B E} \mathcal{H}^{C F} \\
& -\frac{1}{4} f_{B C}^{A} f_{A D}^{B} \mathcal{H}^{C D}-2 \eta_{A} \partial_{B} \mathcal{H}^{A B}+4 \eta_{A} \mathcal{H}^{A B} \partial_{B} d-\eta_{A} \eta_{B} \mathcal{H}^{A B} .
\end{aligned}
$$

The anzats in (4.35) does not yield any deformation in the GDFT action of the RR sector, as it is $F$ and not $\chi$, which is twisted. As a result one ends up with the following action

$$
S_{\mathrm{GDFT}}=v \int d x d \tilde{x}\left(e^{-2 d}\left(\mathcal{R}+\mathcal{R}_{f}\right)+\frac{1}{4}\left\langle\not \partial \chi, C^{-1} \mathbb{S} \not \partial \chi\right\rangle\right)
$$

where $v$ is defined as

$$
v=\int d^{d} Y e^{-2 \rho(Y)} .
$$

Explicit form of the fluxes that determine $\mathcal{R}_{f}$ will be presented in the next subsection. The second term in (4.39) is the usual action for the RR sector of DFT of Type II strings and does not depend on the fluxes, as the duality twisted anzats has been imposed on the spinor field $F$ (which encode the RR fluxes), and not on the spinor field $\chi$ (which encodes the modified gauge potentials). Recall that the relation between the two is as in (2.15). If it were the field $\chi$ which had been twisted, then the DFT action of the RR sector would also be deformed in a way determined by the fluxes. It was shown in [54] that the Lagrangian in this case is of the same form as (4.4), except that $\not \partial$ should be replaced with $\not$. Although we will not need this deformed action in this paper, we will need and present the explicit form of $\not$ in subsection 4.4.2, where we will discuss the field equations arising from (4.39).

\subsubsection{Fluxes, dual fluxes and the $O(d, d)$ invariance of GDFT}

The fluxes that determine the deformation in the NS-NS sector are defined as below [53]

$$
f_{A B C}=3 \Omega_{[A B C]}, \quad \eta_{A}=\partial_{M}\left(U^{-1}\right)_{A}^{M}-2\left(U^{-1}\right)_{A}^{M} \partial_{M} \sigma
$$

where $\sigma$ is as in (4.36) and

$$
\Omega_{A B C}=-\left(U^{-1}\right)_{A}^{M} \partial_{M}\left(U^{-1}\right)_{B}^{N} U_{N}^{D} \eta_{C D}
$$

Note that $\Omega_{A B C}$ are antisymmetric in the last two indices: $\Omega_{A B C}=-\Omega_{A C B}$. We also make the following definition

$$
f_{A}=-\partial_{M}\left(U^{-1}\right)_{A}^{M}=\Omega_{A C}^{C}
$$

The constraints that should be obeyed by the twist matrices are as follows:

$$
\begin{aligned}
\partial^{P}\left(U^{-1}\right)_{A}^{M} \partial_{P} g(X) & =0, \\
\left(U^{-1}\right)_{A}^{M} \partial_{M} g(X) & =\partial_{A} g(X),
\end{aligned}
$$

where $g$ is any of the DFT fields $(\mathcal{H}, \mathbb{S}, \chi)$.

The DFT action of the NS-NS sector is manifestly $O(d, d)$ invariant. So, if $h$ is a constant $O(d, d)$ matrix, then

$$
\mathcal{R}[\mathcal{H}, d, \partial]=\mathcal{R}\left[h^{t} \mathcal{H} h, d, \hat{\partial}\right] .
$$


We have inserted $\partial$ and $\hat{\partial}$ in the arguments of $\mathcal{R}$ to emphasize that the derivatives $\partial_{M}$ on the left hand side should be replaced by $\hat{\partial}_{N} \equiv h_{N}^{M} \partial_{M}$ on the right hand side. On the other hand, the DFT action of the RR sector is $\operatorname{Spin}^{+}(d, d)$ invariant [38]. Therefore, the field equations that arise from varying the DFT action of the NS-NS sector with respect to the generalized metric and the generalized dilaton are $O(d, d)$ covariant, whereas the field equations obtained by varying the DFT action of the RR sector with respect to the spinor field $\chi$ or the spinor field $\mathbb{S}$ are covariant under the subgroup $\operatorname{Spin}^{+}(d, d)$ of $\operatorname{Spin}(d, d)$. This point will be important in section 4.4 , see equations (4.94), (4.95). $\operatorname{Pin}(d, d)$ elements that do not lie in this subgroup act as dualities rather than invariances, as we will discuss in more detail in the next section. The $O(d, d)$ invariance of the generalized scalar curvature $\mathcal{R}$ also extends to $\mathcal{R}_{f}$, provided that we treat the fluxes $f_{A B C}$ as spurious generalized tensors, which also transform under $O(d, d)$. So, if we define

$$
\hat{f}^{A B C}=h_{D}^{A} h_{E}^{B} h_{F}^{C} f^{D E F}, \quad h \in O(d, d)
$$

then it is easily shown that

$$
\mathcal{R}_{\hat{f}}\left[h^{t} \mathcal{H} h, d, \hat{\partial}\right]=\mathcal{R}_{f}[\mathcal{H}, d, \partial]
$$

If the twist matric $h^{M}{ }_{N}$ satisfy the consistency condition (4.45) so that $\hat{\partial}_{M}=\partial_{M}$ acting on the fields $\mathcal{H}(x)$ and $d(x)$, then we simply have

$$
\begin{aligned}
\mathcal{R}\left[h^{t} \mathcal{H} h, d\right] & =\mathcal{R}[\mathcal{H}, d] \\
\mathcal{R}_{\hat{f}}\left[h^{t} \mathcal{H} h, d\right] & =\mathcal{R}_{f}[\mathcal{H}, d] .
\end{aligned}
$$

At this point we find it useful to introduce dual fluxes and dual DFT fields. Let us pick up $h=J$ in (4.47), where $J$ is the matrix obtained by embedding the $d \times d$ matrix $J_{d}$ below in $O(10,10)$ as in $(2.5)$ :

$$
J_{d}=\left(\begin{array}{cc}
0 & 1_{d} \\
1_{d} & 0
\end{array}\right) .
$$

In this particular case, we call the resulting flux the dual flux, and we denote it by $\bar{f}^{A B C}$ for this particular case. That is,

$$
\bar{f}^{A B C}=J_{D}^{A} J_{E}^{B} J_{F}^{C} f^{D E F}
$$

Note that, due to complete antisymmetry of $f_{A B C}$ in its indices, the only independent blocks of $f_{A B C}$ out of the 8 possible combinations are $f_{J K}^{I}, f_{I J K}, f_{K}^{I J}$ and $f^{I J K}, I=1, \cdots, d$. It is customary to call these the geometric flux, the H-flux, the Q-flux and the R-flux, respectively [36]. Obviously, the geometric flux, H-flux, Q-flux and R- flux components of $f$ is replaced by Q-flux, R-flux, geometric flux and H-flux components in $\bar{f}$, respectively. This is the reason why we call the flux $\bar{f}_{A B C}$ the dual flux of $f_{A B C}$. Now, taking $h=J$ in (4.46) and (4.48), we get

$$
\mathcal{R}[\overline{\mathcal{H}}, d, \bar{\partial}]=\mathcal{R}[\mathcal{H}, d, \partial], \quad \text { and } \quad \mathcal{R}_{\bar{f}}[\overline{\mathcal{H}}, d, \bar{\partial}]=\mathcal{R}_{f}[\mathcal{H}, d, \partial]
$$


where we have defined

$$
\overline{\mathcal{H}}=J^{t} \mathcal{H} J, \quad \bar{\partial}_{N}=J^{M}{ }_{N} \partial_{M} .
$$

The DFT field $\overline{\mathcal{H}}$ we defined above has been called the dual generalized metric, in [38]. ${ }^{9}$ Also note that we have $\partial_{i}=\tilde{\bar{\partial}}^{i}, \tilde{\partial}^{i}=\bar{\partial}_{i}$, that is the standard and dual derivatives have been swapped in $\partial$ and $\bar{\partial}$. For future reference, we also define the dual spinor fields $\bar{F}$ and $\bar{\chi}$ as in [38]:

$$
\bar{F}=e^{-\bar{B}} C e^{B} F, \quad \bar{\chi}=C \chi .
$$

It is easily checked that $\bar{F}=e^{-\bar{B} \not \partial \bar{\chi}}$. Here, $\bar{B}$ is the B-field associated with the dual generalized metric $\overline{\mathcal{H}}$.

At this point, it is natural to ask the relation of the twist matrix $\bar{U}$ associated with the fluxes $\bar{f}$ to the twist matrix $U$ associated with the fluxes $f$. One can easily show that the relation is $\bar{U}=J U$. Then, $\bar{S}= \pm S C$, where $\rho(\bar{S})=\bar{U}^{-1}$ and $\rho(S)=U^{-1}$. Indeed, it can easily be shown that

$$
\begin{aligned}
\bar{\Omega}_{A B C} & =-\left(\bar{U}^{-1}\right)_{A}^{M} \partial_{M}\left(\bar{U}^{-1}\right)_{B}^{N} \bar{U}_{N}^{D} \eta_{C D} \\
& =-J_{A}^{D} J_{B}^{E} J_{C}^{F}\left(U^{-1}\right)_{D}^{M} \partial_{M}\left(U^{-1}\right)_{E}^{N} U_{N}^{G} \eta_{F G} \\
& =J_{A}^{D} J_{B}^{E} J_{C}^{F} \Omega_{D E F} .
\end{aligned}
$$

For future reference, we also consider the fluxes associated with the twist matrices $\breve{U}=U J$ and $\breve{S}= \pm C S$, with $\rho(\breve{S})=\breve{U}^{-1}$. One can easily see that the fluxes associated with $\breve{U}$ are exactly the same as the fluxes associated with $U$, except for the fact that all standard/dual derivatives in the computation of $f$ should be replaced with dual/standard derivatives in the computation of $\breve{f}$. More precisely, we have

$$
\begin{aligned}
\breve{\Omega}_{A B C} & =-\left(\breve{U}^{-1}\right)_{A}^{M} \partial_{M}\left(\breve{U}^{-1}\right)_{B}^{N} \breve{U}_{N}^{D} \eta_{C D} \\
& =-\left(U^{-1}\right)_{A}^{M} \bar{\partial}_{M}\left(U^{-1}\right)_{B}^{N} U^{D}{ }_{N} \eta_{C D} \\
& =-\left(U^{-1}\right)_{A}^{M} \partial^{M}\left(U^{-1}\right)_{B}^{N} U^{D}{ }_{N} \eta_{C D} .
\end{aligned}
$$

\subsubsection{Fluxes associated with the NATD matrix}

Let us now compute the fluxes associated with the matrices $L(\theta)$ in $(4.14)$ and $T_{\mathrm{NATD}}(\nu)$ in (3.20). Note that the condition (4.45) is trivially satisfied both by $L(\theta)$ and $T_{\mathrm{NATD}}(\nu)$, as they are constructed by embedding $O(d, d)$ matrices in $O(10,10)$ as in $(2.5)$ and the $d$ coordinates on which $O(d, d)$ acts are not included in the $x$ coordinates of the fields $\mathcal{H}(x), d(x), \chi(x)$ and $\mathbb{S}(x)$. In the computation, the coordinates on which the twist matrices depend are regarded as the standard coordinates and not the winding type ones. To be more precise, the $\theta$ coordinates of the geometric twist matrix $L(\theta)$ are the standard space coordinates for the fields in the supergravity background before the dualisation. Then, after applying the NATD matrix $T_{\mathrm{NATD}}(\nu)$ on the untwisted fields, we end up with a set of fields, which now depend on the coordinates $(x, \nu)$. For the fields after dualisation, it is

\footnotetext{
${ }^{9}$ Note that this is just a field redefinition so there is no transformation on the coordinates. For more details, see [38].
} 
now these coordinates $(x, \nu)$ that are identified with the space-time coordinates. We start by expanding the formula given in (4.41):

$$
\begin{aligned}
\Omega_{A B C} & =-\left(U^{-1}\right)_{A}^{\mu} \partial_{\mu}\left(U^{-1}\right)_{B}^{N} U_{N}^{D} \eta_{C D}-\left(U^{-1}\right)_{\mu A} \tilde{\partial}^{\mu}\left(U^{-1}\right)_{B}^{N} U^{D}{ }_{N} \eta_{C D} \\
& =-\left(U^{-1}\right)^{i}{ }_{A} \partial_{i}\left(U^{-1}\right)_{B}^{N} U^{D}{ }_{N} \eta_{C D} \\
& =-\left(U^{-1}\right)^{i}{ }_{A} \partial_{i}\left(U^{-1}\right)_{B}^{j} U_{j}^{D} \eta_{C D}-\left(U^{-1}\right)^{i}{ }_{A} \partial_{i}\left(U^{-1}\right)_{j B} U^{D j} \eta_{C D} .
\end{aligned}
$$

In passing from the first line to the second line, we used the fact that the twist matrix has no dependence on the winding type coordinates so that all $\tilde{\partial}^{\mu}$ derivatives are zero and that they depend only on the isometry coordinates so that $\partial_{m}\left(U^{-1}\right)_{B}^{N}=0$ (recall that $\mu=(i, m)$, see appendix A.)

When $U^{-1}=T_{\text {NATD }}(\nu)$ in $(3.20)$ we have

$$
\begin{array}{lll}
\left(T_{\mathrm{NATD}}\right)_{i}{ }^{I}=0, & \left(T_{\mathrm{NATD}}\right)_{i I}=\delta_{i I}, & \left(T_{\mathrm{NATD}}\right)^{i I}=\delta^{i I}, \quad\left(T_{\mathrm{NATD}}\right)^{i}{ }_{I}=\theta_{I}^{i}, \\
\left(T_{\mathrm{NATD}}\right)_{m}^{a}=\delta_{m}^{a}, & \left(T_{\mathrm{NATD}}\right)_{a}^{m}=\delta_{a}^{m}, & \left.\left(T_{\mathrm{NATD}}\right)_{m}^{I}=\left(T_{\mathrm{NATD}}\right)_{I}^{m}=\left(T_{\mathrm{NATD}}\right)_{i}{ }^{a}=\left(T_{\mathrm{NATD}}\right)^{i}{ }_{a}=0.59\right)
\end{array}
$$

where we have defined

$$
\theta^{i}{ }_{I}=\delta^{i J} C_{I J}^{K} \nu_{K}
$$

so that the indices match. Plugging these in the formula we find that the only non-vanishing components are

$$
\Omega_{J K}^{I}=-C_{J K}^{I}, \quad \Omega_{I J K}=C_{L I}^{H} C_{J K}^{L} \nu_{H} .
$$

These give rise to the following fluxes

$$
\begin{aligned}
& f_{I J}^{K}=\Omega_{I J}{ }^{K}+\Omega_{J}{ }_{I}+\Omega_{I J}^{K}=-C_{I J}{ }^{K} \\
& f_{I J K}=\Omega_{I J K}+\Omega_{J K I}+\Omega_{K I J}=\frac{1}{2} C_{L[I}^{H} C_{J K]}^{L} \nu_{H}=0,
\end{aligned}
$$

where the last equality follows from the Jacobi identity.

Now, let us compute the fluxes associated with the geometric twist matrix (4.14) so that $U^{-1}=L(\theta)$. In this case the only non-vanishing flux is the geometric flux $f_{I J}{ }^{K}=\Omega_{I J}^{K}-\Omega_{J I}^{K}\left(\right.$ since $\left.\Omega_{I J}^{K}=0\right)$ :

$$
f_{I J}{ }^{K}=-l_{I}^{i} \partial_{i} l^{j}{ }_{J} l_{j}^{K}+l^{i}{ }_{J} \partial_{i} l_{I}^{j} l_{j}^{K}=-C_{I J}{ }^{K} .
$$

This follows from the fact that $\sigma^{I}=l^{I} d \theta^{i}$ are left-invariant one-forms and as such they satisfy

$$
d \sigma^{I}=-\frac{1}{2} C_{I J}^{K} \sigma^{J} \wedge \sigma^{K}
$$

The fluxes associated with $T_{\mathrm{NATD}}$ and $L$ are exactly the same. This will be the key point in proving that NATD is a solution generating transformation for DFT. 


\subsection{Comparing the field equations of DFT and GDFT}

In the previous subsection, we studied GDFT, which is obtained from Scherk-Schwarz reduction of DFT. The Scherk-Schwarz anzats is known to give rise to a consistent dimensional reduction, meaning that any solution of the field equations of the resulting theory can be uplifted to a solution of the higher dimensional field equations $[49,50]$. In our case this implies that any solution of the field equations of GDFT can be uplifted to a solution for DFT. Conversely, the field equations of DFT will reduce to the field equations of GDFT and hence, given a solution of DFT for which the dependence of the fields on the doubled coordinates is separated as in (4.34), (4.35) and (4.36), the untwisted fields $\mathcal{H}(x), F(x), d(x)$ will form a solution of the GDFT equations, where the fluxes in the GDFT action is determined by the twist matrix $U(Y)$.

This straightforward argument should be discussed in more detail, mainly for two reasons. Firstly, the anzats in (4.34), (4.35) and (4.36) is not exactly the Scherk-Scwarz anzats which gives a consistent dimensional reduction to GDFT, due to the difference in the anzats for RR fields. The correct anzats would have been

$$
\chi(x, Y)=e^{-\sigma(Y)} S(Y) \chi(x)
$$

which gives rise to a deformation of the $\mathrm{RR}$ sector, as well. Although the field $\chi$ appears in the DFT action only through its field strength $F=e^{-B \not \partial \chi}$, it has a bare appearance in the gauge transformation rules and hence a consistent reduction should involve an anzats for the field $\chi \cdot{ }^{10}$ However, at the level of equations of motion, this raises no problem since the field $\chi$ never appears in the field equations without a derivative. As a second important point, the real duality group for DFT is $\operatorname{Spin}^{+}(d, d)$ and hence only a twist matrix in this subgroup of $\operatorname{Spin}(d, d)$ can give a consistent reduction. This point is particularly important for us, as the NATD matrix in (3.20) is not in $\operatorname{Spin}^{+}(d, d)$. However, as discussed in [38], although the $\operatorname{Pin}(d, d)$ transformations which are not in this subgroup are not invariances of DFT, they act as duality transformations. This is also true at the level of field equations. In order to clarify these points, we will discuss below the relationship between the field equations of DFT and of GDFT in more detail.

\subsubsection{Field equations for the generalized dilaton field}

The field equations obtained by varying the DFT action with respect to the generalized dilaton field is [34]

$$
\mathcal{R}=0,
$$

where $\mathcal{R}$ is as in (4.7). If we plug in (4.34) and (4.36) in (4.65), we obtain

$$
\mathcal{R}+\mathcal{R}_{f}=0
$$

as was shown in [53]. The form of $\mathcal{R}_{f}$ was given in (4.38). It can be easily shown that this is the field equation obtained by varying (4.39) with respect to the generalized dilaton field. Therefore, a set of DFT fields whose dependence on the coordinates is separated

\footnotetext{
${ }^{10}$ Duality twisted reduction of DFT with the anzats (4.64) was studied in [54].
} 
as in (4.34), (4.35) and (4.36) will satisfy the generalized dilaton field equations of DFT if and only if the untwisted fields $\mathcal{H}(x), d(x)$ satisfy the generalized dilaton equations for the GDFT, where the fluxes $f_{A B C}$ which determine the deformation are determined by the twist matrix $U$.

\subsubsection{Field equations for the spinor field $\chi$}

The field equation for the spinor field $\chi$ is [38]

$$
\not \partial(\mathcal{K} \not \partial \chi)=0,
$$

which is to be supplemented with the duality constraint

$$
\not \partial \chi=-\mathcal{K} \not \partial \chi
$$

In terms of the field $F=e^{-B} \not \partial \chi$ the equation and the duality constraint becomes:

$$
\begin{aligned}
\not \partial\left(\mathcal{K} e^{B} F\right) & =0 \\
F & =-e^{-B} \mathcal{K} e^{B} F .
\end{aligned}
$$

Imposing the duality constraint on the field equation we get

$$
\not \partial\left(e^{B} F\right)=0 .
$$

Let us plug in (4.34) and (4.35) in (4.70). We immediately see that the duality constraint is satisfied by $\mathcal{H}(x, Y), F(x, Y)$ if and only if the same duality constraint is satisfied by $F(x), \mathcal{H}(x)$ :

$$
F(x)=-e^{-B(x)} \mathcal{K}(x) e^{B(x)} F(x) .
$$

On the other hand, plugging (4.34) and (4.35) into the field equation (4.69) we get

$$
\not \partial\left(e^{-\sigma(Y)} S(Y) \mathcal{K}(x) e^{B(x)} F(x)\right)=0 .
$$

We plug the duality constraint (4.72) in (4.73) to get (recall that $\not \partial=\Gamma^{M} \partial_{M}$ ):

$$
e^{-\sigma(Y)} S(Y)\left\{S^{-1}(Y) \Gamma^{M} \partial_{M}(S(Y)-\sigma(Y))+\not \partial\right\}\left(e^{B(x)} F(x)\right)=0 .
$$

Now we use the following facts [54]:11

$$
\begin{aligned}
S^{-1} \Gamma^{M} S & =\left(U^{-1}\right){ }_{A}^{M} \Gamma^{A} \\
\Gamma^{A}\left(U^{-1}\right){ }_{A} S^{-1} \partial_{M} S & =\frac{1}{4} \Omega_{A B C} \Gamma^{A} \Gamma^{B} \Gamma^{C} \\
& =\frac{1}{12} f_{A B C} \Gamma^{A} \Gamma^{B} \Gamma^{C}-\frac{1}{2} f_{B} \Gamma^{B},
\end{aligned}
$$

where $U=\rho\left(S^{-1}\right)$. Using these one can show easily that the equation (4.74) is equivalent to

$$
\not\left(e^{B(x)} F(x)\right)=0
$$

\footnotetext{
${ }^{11}$ We proved the identity (4.76) in [54] for $S \in \operatorname{Spin}^{+}(d, d)$. It can be easily shown that it also holds for elements of $S \in \operatorname{Spin}(d, d)$ of the form $S=C S^{+}$and $S=S^{+} C$, where $S^{+} \in \operatorname{Spin}^{+}(d, d)$.
} 
where the Dirac operator $\not$ is defined as (see [54])

$$
\not \nabla \equiv \not \partial+\frac{1}{12} f_{A B C} \Gamma^{A} \Gamma^{B} \Gamma^{C}-\frac{1}{2} \eta_{B} \Gamma^{B} .
$$

As a result, we conclude that the fields (4.34) and (4.35) form a solution for the field equation (4.71) if and only if the untwisted fields $F(x), \mathcal{H}(x)$ satisfy $(4.77) .{ }^{12}$

\subsubsection{Field equations for the generalized metric $\mathcal{H}_{M N}$}

The field equations obtained from varying the DFT action with respect to the generalized metric $\mathcal{H}_{M N}$ is [34, 38]:

$$
R_{M N}+e^{-2 \phi} \Xi_{M N}=0,
$$

where

$$
\begin{aligned}
\Xi^{M N} & =\frac{1}{16} \mathcal{H}_{P}^{(M}\left\langle\not \partial \chi, \Gamma^{N) P} \mathcal{K} \not \partial \chi\right\rangle=\frac{1}{16} \mathcal{H}_{P}^{(M}\left\langle e^{B} F, \Gamma^{N) P} \mathcal{K} e^{B} F\right\rangle \\
& =-\frac{1}{16} \mathcal{H}_{P}^{(M}\left\langle F, e^{-B} \Gamma^{N) P} e^{B} F\right\rangle .
\end{aligned}
$$

The first term in (4.79) comes from the variation of the GDFT action of the NS-NS sector, and the variation of the GDFT action of the RR sector gives the second term. In passing to the second line in (4.80), we used the invariance property of Mukai pairing under $\operatorname{Spin}^{+}(d, d),{ }^{13}$ (which $e^{-B}$ is an element of), and we also imposed the duality constraint $\not \partial \chi=-\mathcal{K} \not \partial \chi$. Here, $\Gamma^{M N}$ is defined as $\Gamma^{P Q} \equiv \frac{1}{2}\left[\Gamma^{P}, \Gamma^{Q}\right]$. Let us plug in the set of fields in (4.34), (4.35), (4.36) into these equations. Consider first the following expression:

$$
\left(U^{-1}\right)_{A}^{M} R_{M N}[\mathcal{H}(x, Y), d(x, Y)]\left(U^{-1}\right)_{B}^{N} .
$$

We emphasize again that $R_{M N}[\mathcal{H}(x, Y), d(x, Y)]$ is obtained by varying $e^{-2 d} \mathcal{R}$ with respect to $\mathcal{H}^{M N}$ and then plugging in $\mathcal{H}(x, Y)$. Now compare the expression in (4.82) with the variation of the GDFT action of the NS-NS sector (which is obtained by plugging in $\mathcal{H}(x, Y)$ in $\mathcal{R}$ first) with respect to $\mathcal{H}^{A B}$. Comparing term by term, one sees that the two give the same result. Then, we have

$$
e^{2 d}\left(U^{-1}\right)_{A}^{M} R_{M N}[\mathcal{H}(x, Y), d(x, Y)]\left(U^{-1}\right)_{B}^{N}=\frac{\delta\left(e^{2 d}\left(\mathcal{R}+\mathcal{R}_{f}\right)\right)}{\delta \mathcal{H}^{A B}} .
$$

So, if we define

$$
e^{2 d} R_{f}^{A B}=\frac{\delta\left(e^{2 d} \mathcal{R}_{f}\right)}{\delta \mathcal{H}_{A B}}
$$

\footnotetext{
${ }^{12}$ Note that (4.77), which is equivalent to $\not \nabla(\not \chi \chi(x))=0$ is not the field equation obtained from varying (4.39) with respect to the spinor field $\chi$, which would have given $\not \partial(\not \partial \chi(x))=0$. It is not the field equation obtained from varying the GDFT action of the RR sector obtained in [54] through a duality twisted ansazt on $\chi$ (rather than $F$ ) either, which would have yielded $\not(\not \chi(x))=0$. Note that both of these equations are satisfied automatically due to nilpotency of $\not \partial$ and $\not$.

${ }^{13}$ The Mukai pairing satisfies

$$
\left\langle S . \phi_{1}, S . \phi_{2}\right\rangle= \pm\left\langle\phi_{1}, \phi_{2}\right\rangle, \quad S \in \operatorname{Spin}^{ \pm}(d, d) .
$$


then we have

$$
R^{M N}[\mathcal{H}(x, Y), d(x, Y)]=\left(U^{-1}\right)_{A}^{M}\left(R^{A B}[\mathcal{H}(x), d(x)]+R_{f}^{A B}[\mathcal{H}(x), d(x)]\right)\left(U^{-1}\right)^{N}{ }_{B} .
$$

Now, we plug in $\Xi^{M N}$ the fields $\mathcal{H}, \mathcal{K}, F, B$, whose dependence on the coordinates $(x, Y)$ is separated as in (4.34), (4.35), (4.36). If we use the invariance property of the Mukai pairing and the following identity

$$
S^{-1} \Gamma^{M N} S=\Gamma^{A B}\left(U^{-1}\right)_{A}^{M}\left(U^{-1}\right)_{B}^{N},
$$

along with

$$
\mathcal{H}_{P}^{M}(x, Y)=\left(U^{-1}\right){ }_{A}^{M} U_{P}^{B} \mathcal{H}_{B}^{A},
$$

we obtain

$$
\begin{aligned}
& -\frac{1}{16}\left(U^{-1}\right)_{A}^{M}(Y)\left(U^{-1}\right)_{B}^{N}(Y) \mathcal{H}_{C}^{(A}(x)\left\langle S(Y) e^{B(x)} F(x), S(Y) \Gamma^{B) C} e^{B(x)} F(x)\right\rangle \\
& =\mp \frac{1}{16}\left(U^{-1}\right)_{A}^{M}(Y) \mathcal{H}_{C}^{(A}(x)\left\langle e^{B(x)} F(x), \Gamma^{B) C} e^{B(x)} F(x)\right\rangle\left(U^{-1}\right)_{B}^{N}(Y), \quad S \in \operatorname{Spin}^{ \pm}(10,10) .
\end{aligned}
$$

Therefore, we have found that

$$
e^{-2 d(x, Y)} \Xi^{M N}[\mathcal{H}(x, Y), F(x, Y), d(x, Y)]=\left(U^{-1}\right)^{M}{ }_{A}(Y) e^{-2 d(x)} \Xi^{A B}[\mathcal{H}(x), F(x), d(x)]\left(U^{-1}\right)^{N}(Y),
$$

if the twist matrix $S(Y)$ is in $\operatorname{Spin}^{+}(d, d)$.

Now consider the case when $S$ is not in $\operatorname{Spin}^{+}(d, d)$. We assume that it is of the form $S(Y)=S_{1}(Y) C$, where $S_{1} \in \operatorname{Spin}^{+}(d, d)$ and $C$ is the charge conjugation element satisfying $\rho(C)=J$. (This is the case for the twist matrices that determines our NATD fields. Recall that $S_{\mathrm{NATD}}(\nu)=S_{\beta}(\nu) C$ and $S_{\beta} \in \operatorname{Spin}^{+}(d, d)$.) In this case only the $S_{1}(Y)$ factor can be dropped in passing from the first line to the second line in (4.88) and we end up with

$$
\begin{aligned}
& -\frac{1}{16}\left(U^{-1}\right)_{A}^{M}(Y)\left(U^{-1}\right)_{B}^{N}(Y) \mathcal{H}_{D}^{(A}(x)\left\langle C e^{B(x)} F(x), C \Gamma^{B) D} e^{B(x)} F(x)\right\rangle \\
& =-\frac{1}{16}\left(U_{1}^{-1}\right)_{A}^{M}(Y)\left(U_{1}^{-1}\right)_{B}^{N}(Y) \overline{\mathcal{H}}_{D}^{(A}(x)\left\langle C e^{B(x)} F(x), \Gamma^{B) D} C e^{B(x)} F(x)\right\rangle
\end{aligned}
$$

where $\rho\left(S_{1}\right)=U_{1}$ and $\overline{\mathcal{H}}=J^{T} \mathcal{H} J$ is the dual generalized metric we defined in (4.54). Note that in writing the second line above we used

$$
\left(U^{-1}\right)^{T} \mathcal{H} U^{-1}=\left(\left(U_{1} J\right)^{-1}\right)^{T} \mathcal{H}\left(U_{1} J\right)^{-1}=\left(U_{1}^{-1}\right)^{T} J^{T} \mathcal{H} J U_{1}^{-1}=\left(U_{1}^{-1}\right)^{T} \overline{\mathcal{H}} U_{1}^{-1} .
$$

Also recalling the definition of the dual spinor field $\bar{F}$ in (4.55) we see that (4.91) above can be written in terms of the dual fields and we have:

$$
\Xi^{M N}[\mathcal{H}(x, Y), F(x, Y), d(x, Y)]=\left(U_{1}^{-1}\right)_{A}^{M}(Y) \Xi^{A B}[\overline{\mathcal{H}}(x), \bar{F}(x), d(x)]\left(U_{1}^{-1}\right)_{B}^{N}(Y) .
$$

Let us now try and write the $R_{M N}$ part of the field equation in terms of the dual generalized metric $\overline{\mathcal{H}}$, as well. For this we need to observe that this piece of the field equation is $O(d, d)$ covariant:

$$
\begin{aligned}
R^{A B}[\mathcal{H}(x)] & =R^{A B}[J \overline{\mathcal{H}} J]=J R^{A B}[\overline{\mathcal{H}}(x), d] J \\
R_{f}^{A B}[\mathcal{H}(x), d] & =R_{f}^{A B}[J \overline{\mathcal{H}}(x) J d]=J R_{\bar{f}}^{A B}[\overline{\mathcal{H}}(x), d] J
\end{aligned}
$$


where $\bar{f}$ is the dual flux we defined in (4.52). These follow directly from the $O(d, d)$ covariance of $\mathcal{R}$ and $\mathcal{R}_{f}$, see (4.53). Recall that $J$ is obtained by embedding the $O(d, d)$ matrix $J_{d}$ in $O(10,10)$ as in $(2.5)$. Hence, it acts non-trivially only on the isometry directions and acts on the partial derivatives with respect to $x$ coordinates as an identity transformation. Therefore, we have

$$
J^{M}{ }_{A} \partial_{M} g(x)=\partial_{A} g(x),
$$

where $g(x)$ denotes any of the untwisted fields $\mathcal{H}(x), F(x), d(x)$ or $\mathbb{S}(x)$. So, we have $\bar{\partial}=\partial$ in (4.53). As a result, using (4.92), we can rewrite (4.85) as:

$$
\begin{aligned}
R^{M N}[\mathcal{H}(x, Y), d(x, Y)] & =\left(U^{-1}\right)_{A}^{{ }_{A}}\left(R^{A B}[\mathcal{H}(x), d(x)]+R_{f}^{A B}[\mathcal{H}(x), d(x)]\right)\left(U^{-1}\right)_{B}^{N} \\
& =\left(U_{1}^{-1}\right)^{M}{ }_{A} R^{A B}[\overline{\mathcal{H}}(x), d(x)]+R_{\bar{f}}^{A B}[\overline{\mathcal{H}}(x), d(x)]\left(U_{1}^{-1}\right)^{N}{ }_{B} .
\end{aligned}
$$

To recap, we have obtained the following: for $S \in \operatorname{Spin}^{+}(d, d)$ with $\rho(S)=U^{-1}$, equation (4.79) is satisfied by the fields $\mathcal{H}(x, Y), \mathbb{S}(x, Y), F(x, Y)$ and $d(x, Y)$ if and only if the untwisted fields satisfy the following GDFT equation:

$$
R^{A B}[\mathcal{H}(x), d(x)]+R_{f}^{A B}[\mathcal{H}(x), d(x)]+e^{-2 d(x)} \Xi^{A B}[\mathcal{H}(x), F(x), d(x)]=0 .
$$

Here the fluxes $f$ in $R_{f}$ are produced by the twist matrix $U$.

On the other hand, if $S=S_{1} C$ with $S_{1} \in \operatorname{Spin}^{+}(d, d)$ and $\rho\left(S_{1}\right)=U_{1}^{-1}$ so that $U=J U_{1}$ we can make the following statement. The twisted fields $\mathcal{H}(x, Y), \mathbb{S}(x, Y), F(x, Y)$ and $d(x, Y)$ satisfy equation (4.79) if and only if the untwisted dual fields $\overline{\mathcal{H}}(x), \bar{F}(x)$ and $d(x)$ satisfy

$$
R^{A B}[\overline{\mathcal{H}}(x), d(x)]+R_{\bar{f}}^{A B}[\overline{\mathcal{H}}(x), d(x)]+e^{-2 d(x)} \Xi^{A B}[\overline{\mathcal{H}}(x), \bar{F}(x), d(x)]=0 .
$$

Here, the fluxes $\bar{f}$ in $R_{\bar{f}}$ are fluxes dual to $f$, and the fluxes $f$ are produced by the twist matrix $U$.

\subsection{NATD fields as a solution of DFT in the supergravity frame}

We are now ready to prove our claim that NATD is a solution generating transformation for DFT, that is, the fields (4.30), (4.31), (4.32) corresponding to the NATD background solve DFT equations. As we discussed before, this immediately proves that the NATD fields form a solution of Type II supergravity, if we identify $(x, \nu)$ with standard spacetime coordinates. This is because in the frame $\tilde{\partial}^{\mu}=0$ the DFT equations will reduce to Type IIA or Type IIB equations depending on the fixed chirality of $\chi \cdot^{14}$

In the previous section, we saw that the fields $\mathcal{H}(x, Y), F(x, Y), \mathbb{S}(x, Y)$ and $d(x, Y)$ in (4.34)-(4.36) satisfy the field equations of DFT if and only if the untwisted fields $\mathcal{H}(x), F(x), \mathbb{S}(x)$ and $d(x)$ satisfy the field equations of the GDFT determined by the fluxes associated with the twist matrix $U$. This implies the following: suppose that we know the fields $\mathcal{H}(x, Y), F(x, Y), \mathbb{S}(x, Y)$ and $d(x, Y)$ satisfy the field equations of DFT. Then, the

\footnotetext{
${ }^{14}$ We still assume that the duality group is unimodular. If not, the dilaton field is forced to have a linear dependence on winding type coordinates taking the NATD background out of the supergravity frame. We will discuss this in section 4.6.
} 
untwisted fields satisfy the field equations of GDFT determined by the fluxes associated with $U$ and $S$. Now, consider another set of fields $\tilde{\mathcal{H}}(x, Z), \tilde{F}(x, Z), \tilde{d}(x, Z)$ obtained by twisting the same fields $\mathcal{H}(x), F(x), d(x)$ by the twist matrices $\tilde{U}(Z)$ and $\tilde{S}(Z)$, where $\tilde{U}$ is also in $\mathrm{SO}^{+}(d, d)$. Suppose also that the fluxes generated by $\tilde{U}(Z)$ and $\tilde{S}(Z)$ are the same as the fluxes generated by $U(Y)$ and $S(Y)$. Since we already know that the untwisted fields satisfy the field equations of GDFT determined by these fluxes, we immediately conclude that the twisted fields $\tilde{\mathcal{H}}(x, Z), \tilde{F}(x, Z), \tilde{d}(x, Z)$ satisfy the field equations of DFT, as well. If the NATD matrix $(3.20)$ were in $\mathrm{SO}^{+}(10,10)$, this argument would immediately imply that the fields (4.30)-(4.32) formed a solution of the DFT equations (4.65), (4.71) and (4.79), since we already know that the untwisted fields $\mathcal{H}(x), d(x), \mathbb{S}(x)$ and $F(x)$ satisfy the GDFT equations (4.66), (4.77) and (4.97). This is known because the fields $\mathcal{H}(x, \theta), \mathbb{S}(x, \theta)$ and $F(x, \theta)$ in $(4.15),(4.18)$ and (4.23) form a solution of the DFT equations (4.65), (4.71) and (4.79) by construction, and the twist matrix $L(\theta)$ in (4.14) generates the same fluxes as the NATD matrix (3.20) does. However, the NATD matrix $T_{\mathrm{NATD}}$ is not in $\mathrm{SO}^{+}(10,10)$. Even in this case, our argument above still holds when we compare the DFT and GDFT equations (4.65) and (4.66) coming from the variation with respect to the generalized dilaton field $d$ and the equations (4.71) and (4.77) coming from the variation with respect to the spinor field $\chi$, since these equations are not just $\mathrm{SO}^{+}(d, d)$ covariant; they are covariant under the full duality group $O(d, d)$. So, the only issue we should discuss is how we compare equations (4.97) and (4.98).

In order to understand this, we look at a generic case in which $U$ is in $\mathrm{SO}^{+}(d, d)$, and $\tilde{U}$ is not. We saw that comparing the generalized metric field equations of DFT and the GDFT is subtle due to the fact that the DFT of the RR sector of Type II strings is invariant only under the subgroup $\operatorname{Spin}^{+}(d, d)$ and $\operatorname{Pin}(d, d)$ transformations that are not in this subgroup must be viewed as dualities and not invariances. In analyzing this case, we found it useful to define the following dual fields, as in [38], which we rewrite here for convenience:

$$
\overline{\mathcal{H}}=J \mathcal{H} J, \quad \bar{F}=e^{-\bar{B}} C e^{B} F .
$$

Recall that $\bar{F}=e^{-\bar{B} \not \partial \bar{\chi}}$, where $\bar{\chi}=C \chi$. It is possible to formulate the DFT action in terms of these dual fields. In fact, it was shown in [38] that the DFT action takes the same form in terms of these dual fields as the action (4.4), provided that we also transform the partial derivatives as $\partial_{i} \leftrightarrow \tilde{\partial}^{i}, i=1, \cdots, d$. We will call this action the dual DFT action. ${ }^{15}$ If the chirality of the spinor field $\chi$ is fixed in such a way that the DFT action reduces to the action of Type IIA/IIB theory in the supergravity frame $\tilde{\partial}^{i}=0$, the dual DFT action reduces to the action of Type IIB/IIA theory in the frame $\partial_{i}=0,[34,38]$. This is when $d$ is odd. If $d$ is even, the chirality of the dual spinor field remains the same, and the dual action reduces to the same Type II action in the frame $\partial_{i}=0 .{ }^{16}$

\footnotetext{
${ }^{15}$ In fact, the DFT action of the RR sector picks up an overall minus sign but so does the duality condition. Hence, when we plug in the duality condition into the field equations, there is no overall minus sign and the form of the field equations are exactly the same both in terms of the original and the dual fields and coordinates.

${ }^{16}$ If the time direction is also dualized, the resulting theory is Type IIA ${ }^{\star}$ or Type IIB $^{\star}$ depending on the chirality, see [38].
} 
Consider a set of fields, which form a solution for the DFT field equations in a certain frame. ${ }^{17}$ Then, the dual fields will satisfy the equations arising from the dual DFT action for the dual fields $\overline{\mathcal{H}}$ and $\bar{F}$. We emphasize again that these equations have exactly the same form as the equations for the original fields, except that the standard derivatives along the directions on which $J_{d}$ acts have been replaced by the winding type derivatives and vice versa. ${ }^{18}$ If the dependence of the fields forming the DFT solution on the coordinates $(x, Y)$ is separated as in (4.34), (4.35), (4.36), then the dependence of the dual fields on these coordinates is also separated in the following way:

$$
\begin{aligned}
\overline{\mathcal{H}}(x, Y) & =\left(\hat{U}^{-1}\right)^{T}(Y) \overline{\mathcal{H}}(x) \hat{U}^{-1}(Y), \quad \overline{\mathcal{K}}(x, Y)=\hat{S}(Y) \overline{\mathcal{K}}(x) \hat{S}^{-1}(Y) \\
\bar{F}(x, Y) & =e^{-\sigma(Y)} e^{-\bar{B}(x, Y)} \hat{S}(Y) e^{\bar{B}(x)} \bar{F}(x), \\
\bar{d}(x, Y) & =\bar{d}(x)+\sigma(Y)
\end{aligned}
$$

where $\hat{S}=C S C^{-1}, \rho(\hat{S})=\hat{U}^{-1}=J U^{-1} J$, and $\bar{F}(x, Y)=e^{-\bar{B}(x, Y)} C \not \partial \chi(x, Y)$ and $\bar{d}=d$. Consider the field equation arising from varying the dual DFT action with respect to the dual generalized metric field and assume that it is satisfied by the dual fields $\overline{\mathcal{H}}(x, Y), \bar{F}(x, Y)$ and $d(x)$ in (4.100)-(4.102). As emphasized above, this equation is exactly of the same form as the generalized metric field equation (4.79) (albeit with $\partial_{i} \leftrightarrow \tilde{\partial}^{i}$ ), which means that we can apply the arguments in section 4.4.3 directly. So, if $S$ is in $\operatorname{Spin}^{+}(d, d)$, so that $\left.\hat{S}=C S C^{-1} \in \operatorname{Spin}^{+}(d, d)\right)$, we find that the twisted dual DFT fields satisfy

$$
R_{M N}[\overline{\mathcal{H}}(x, Y), d(x)]+e^{-2 \phi} \Xi_{M N}[\overline{\mathcal{H}}(x, Y), \bar{F}(x, Y), d(x)]=0
$$

if and only the untwisted dual DFT fields satisfy the following equation

$$
\left(\hat{U}^{-1}\right)_{A}^{M}(Y)\left(\left(R+R_{\hat{f}}\right)^{A B}[\overline{\mathcal{H}}(x), \bar{F}(x), d(x)]+e^{-2 d(x)} \Xi^{A B}[\overline{\mathcal{H}}(x), \bar{F}(x), d(x)]\right)\left(\hat{U}^{-1}\right)_{B}^{N}(Y)=0 .
$$

Since all the $\partial_{i}$ derivatives in (4.103) has been swapped with the winding type derivatives $\tilde{\partial}^{i}$, in calculating the fluxes $\hat{f}$ with the formula (4.41) (with $U=\hat{U}$ ), one should replace $\partial_{i} \leftrightarrow \tilde{\partial}^{i}$. Now remember our discussion in section 4.3.2. From (4.58) we see that the fluxes $\hat{f}$ are produced by the matrix $\breve{U}=\hat{U} J$. Since $\hat{U}=J U J$ we see that the fluxes $\hat{f}$ are the same fluxes as those produced by the twist matrix $\breve{U}=\hat{U} J=J U$, since $J^{2}=I d$. But, according to (4.56) this is just the dual flux $\bar{f}$ to the flux $f$ produced by the twist matrix $U$, that is, $\hat{f}=\bar{f}$. As a result, the equation (4.104) is equivalent to the equation (4.98). This gives

\footnotetext{
${ }^{17}$ For now, we keep the discussion general, but our ultimate goal is to apply the discussion we have here to the fields (4.15), (4.18) and (4.23).

${ }^{18}$ Let us clarify a point that is potentially confusing. When the frame in which the fields satisfy the DFT equations is the supergravity frame (that is, the fields have no dependence on dual coordinates $\tilde{x}$ ), they also form a solution of Type IIA(/IIB) supergravity. Since the dual fields will not belong to the frame $\partial_{i}=0$ in general, they do not necessarily form a solution of Type IIB(/IIA) supergravity. Nevertheless, they are a solution of the field equations of the dual DFT action, and that is all the information we need. In the special case when the isometry group is Abelian, one can pick up coordinates with respect to which the twisted fields will have no dependence on the coordinates $x^{i}, i=1, \cdots, d$ either, so the dual fields will belong to the frame $\partial_{i}=0$. Being a solution of the dual DFT equations, they will hence form a solution of Type IIB(/IIA) supergravity. This is what happens in Abelian T-duality.
} 
us the result that we want: the fact that the fields $\mathcal{H}(x, \theta), F(x, \theta), d(x)$ in (4.15), (4.23) satisfy the DFT equation (4.79) implies that the dual fields $\overline{\mathcal{H}}(x, \theta), \bar{F}(x, \theta), d(x)$ satisfy the dual DFT equation (4.103). As a result, the dual untwisted fields $\overline{\mathcal{H}}(x), \bar{F}(x), d(x)$ satisfy the GDFT equation (4.98), which then implies that the NATD fields $\mathcal{H}(x, \nu), F(x, \nu), d(x)$ in (4.30)-(4.32) satisfy the DFT equation (4.79), as desired.

\subsection{Non-unimodular case: generalized supergravity equations}

So far, we have assumed that the isometry group $G$ is unimodular so that the structure constants $C_{I J}^{K}$ are traceless. When this assumption is relaxed, it is known that the resulting NATD fields form a solution of the GSE, which have recently been introduced in $[18,19]$. Let us see how this situation fits within the framework of DFT.

For simplicity, we assume that the structure constants of the Lie algebra of $G$ have only trace components. Then, the only non-vanishing components of the flux associated with the twist matrix $L$ in (4.15) will be $f_{I}, I=1, \cdots, d$. This contributes to $\eta_{I}$, whose definition is given in (4.41). However, it is well-known that the GDFT action with non-vanishing $\eta_{A}$ is not consistent $[51,53])$. Therefore, the $f_{I}$ part in (4.41) should be compensated by a non-trivial dilaton anzats. A similar situation was also considered [58]. Rewriting (4.41) in components, we see that we need to have

$$
\begin{aligned}
& \eta^{I}=f^{I}-\left(U^{-1}\right)^{M I} \partial_{M} \sigma=0, \\
& \eta_{I}=f_{I}-\left(U^{-1}\right)^{M}{ }_{I} \partial_{M} \sigma=0 .
\end{aligned}
$$

This implies that

$$
\begin{aligned}
\left(U^{-1}\right)^{M I} \partial_{M} \sigma & =f^{I}=0, \\
\left(U^{-1}\right)^{M}{ }_{I} \partial_{M} \sigma & =f_{I}=\text { constant. }
\end{aligned}
$$

When the twist matrix is equal to the NATD matrix (3.20), we can expand these equation by using (4.59) as:

$$
\begin{aligned}
\delta^{i I} \partial_{i} \sigma & =0 \\
\theta^{i}{ }_{I} \partial_{i} \sigma+\delta_{i I} \tilde{\partial}^{i} \sigma & =\text { constant. }
\end{aligned}
$$

As a result, we obtain

$$
\partial_{i} \sigma=0, \quad \tilde{\partial}^{i} \sigma=\text { constant. }
$$

In other words, $\sigma$ is linear in the dual coordinates and does not depend on the standard coordinates. Then, the generalized dilaton field in (4.33) is of the form:

$$
d(x, \tilde{\nu})=d(x)+m_{i} \tilde{\nu}^{i},
$$

where $m_{i}$ are constants.

Appearance of winding type coordinates in the transformed DFT fields means that we are not in the supergravity frame anymore. (Note that, due to the form of the anzats (4.32), the spinor field $F$ also has a dependence on $\tilde{\nu})$. The other DFT fields $\mathcal{H}$ and $\mathbb{S}$ depend only on the space-time coordinates. In the papers [39] and [40], it was shown that the equations of DFT reduce to GSE in such a frame. As a result, the fields in the target space of the NATD model form a solution of GSE, when $G$ is non-unimodular. 


\section{Conclusions and outlook}

In this paper, we studied NATD as a coordinate dependent $O(d, d)$ transformation. The dependence on the coordinates is determined by the structure constants of the Lie algebra of the isometry group $G$. Besides making calculations significantly easier, our approach gives a natural embedding of NATD in Double Field Theory (DFT), a framework which provides an $\mathrm{O}(\mathrm{d}, \mathrm{d})$ covariant formulation for effective string actions [28]-[38] by introducing dual, winding type coordinates. As a result of this embedding, we managed to prove that the NATD fields (both in the NS-NS and the RR sector) solve supergravity equations, when the isometry algebra is unimodular. When the isometry algebra is non-unimodular, we showed that the generalized dilaton field of DFT is forced to have a linear dependence on the winding type coordinates, which implies that the NATD fields solve GSE, in agreement with the literature.

We believe that identifying the $O(d, d)$ matrix that generates the NATD background is important, as it should make it easier to study some properties (such as supersymmetry and integrability) of the NATD backgrounds and their CFT duals, as the relation to the original background is more explicit. On the other hand, our approach also makes it possible to explore the relation between NATD and Yang-Baxter (YB) deformations in detail. Homogoneous YB deformation of an integrable sigma model [59] is determined by the so called R-matrix, which forms a solution of the classical Yang-Baxter equation. In the paper [60], it was conjectured that homogoneos YB models can be obtained by applying NATD to the original background, with respect to an isometry group determined by the Rmatrix. This conjecture was proved in [61] for the case of Principal Chiral Models (PCM) and they extended their work to homogenous YB deformations of more general sigma model than PCM's in [17]. Then, the results of our paper implies that it should be possible to describe YB deformations also as $O(d, d)$ transformations. This approach was also taken in the papers [41, 42], [62]-[65] (see also the papers [66, 67] for a related approach). The methods we have developed in this paper should give a deeper insight on YB deformations and the relation between NATD and YB deformations. We hope to come back to these issues in the near future [68].

\section{Acknowledgments}

We would like to thank E. O Colgain and N.S. Deger for comments and discussions. This work is partially supported by the Turkish Council of Research and Technology (TÜBITAK) through the ARDEB 1001 project with grant number 114F321, in conjunction with the COST action MP1405 QSPACE. We also acknowledge the financial support of Scientific Research Coordination Unit of Istanbul Technical University (ITU BAP) under the project TGA-2018-41742. 


\section{A Index conventions}

Our index conventions are as follows:

$$
\begin{aligned}
& M, N, \cdots: \text { Doubled coordinates; }{ }^{M}=\left(\mu,{ }^{\mu}\right) \\
& A, B, \cdots: \text { Doubled coordinates; }{ }^{A}=\left(\alpha,{ }^{\alpha}\right) \\
& \mu=(i, m), \quad \mu=1, \cdots, 10 ; \quad i=1, \cdots, d, \quad d=\operatorname{dim} G \\
& \alpha=(I, a), \quad \alpha=1, \cdots, 10 ; \quad I=1, \cdots, d
\end{aligned}
$$

According to the embedding rules in (2.5), a twist matrix $T \in O(D, D, R)$, which only twists the $d$ isometry directions is of the following form:

$$
T_{A}^{M}=\left(\begin{array}{cc}
T_{\mu}^{\alpha} & T_{\mu \alpha} \\
T^{\mu \alpha} & T_{\alpha}^{\mu}
\end{array}\right),
$$

with $T_{m}^{a}=\delta_{m}^{a}, T_{a}^{m}=\delta_{a}^{m}, T_{m}^{I}=T_{I}^{m}=T_{i}^{a}=T_{a}^{i}=0$.

Open Access. This article is distributed under the terms of the Creative Commons Attribution License (CC-BY 4.0), which permits any use, distribution and reproduction in any medium, provided the original author(s) and source are credited.

\section{References}

[1] X.C. de la Ossa and F. Quevedo, Duality symmetries from nonAbelian isometries in string theory, Nucl. Phys. B 403 (1993) 377 [hep-th/9210021] [InSPIRE].

[2] E. Alvarez, L. Alvarez-Gaume and Y. Lozano, NonAbelian duality in WZW models, (1994) [INSPIRE].

[3] A. Giveon and M. Roček, On nonAbelian duality, Nucl. Phys. B 421 (1994) 173 [hep-th/9308154] [INSPIRE].

[4] K. Sfetsos, Gauged WZW models and nonAbelian duality, Phys. Rev. D 50 (1994) 2784 [hep-th/9402031] [INSPIRE].

[5] E. Alvarez, L. Álvarez-Gaumé and Y. Lozano, On nonAbelian duality, Nucl. Phys. B 424 (1994) 155 [hep-th/9403155] [INSPIRE].

[6] K. Sfetsos and D.C. Thompson, On non-abelian T-dual geometries with Ramond fluxes, Nucl. Phys. B 846 (2011) 21 [arXiv:1012.1320] [InSPIRE].

[7] Y. Lozano, E. O Colgain, K. Sfetsos and D.C. Thompson, Non-abelian T-duality, Ramond Fields and Coset Geometries, JHEP 06 (2011) 106 [arXiv:1104.5196] [INSPIRE].

[8] G. Itsios, Y. Lozano, E. O Colgain and K. Sfetsos, Non-Abelian T-duality and consistent truncations in type-II supergravity, JHEP 08 (2012) 132 [arXiv:1205.2274] [INSPIRE].

[9] G. Itsios, C. Núnez, K. Sfetsos and D.C. Thompson, Non-Abelian T-duality and the AdS/CFT correspondence:new $N=1$ backgrounds, Nucl. Phys. B 873 (2013) 1 [arXiv:1301.6755] [INSPIRE].

[10] J. Jeong, O. Kelekci and E. O Colgain, An alternative IIB embedding of F(4) gauged supergravity, JHEP 05 (2013) 079 [arXiv:1302.2105] [INSPIRE]. 
[11] K. Sfetsos and D.C. Thompson, New $\mathcal{N}=1$ supersymmetric Ad $S_{5}$ backgrounds in Type IIA supergravity, JHEP 11 (2014) 006 [arXiv:1408.6545] [INSPIRE].

[12] E. Caceres, N.T. Macpherson and C. Núñez, New Type IIB Backgrounds and Aspects of Their Field Theory Duals, JHEP 08 (2014) 107 [arXiv:1402.3294] [INSPIRE].

[13] N.T. Macpherson, C. Núñez, L.A. Pando Zayas, V.G.J. Rodgers and C.A. Whiting, Type IIB supergravity solutions with $A d S_{5}$ from Abelian and non-Abelian T dualities, JHEP 02 (2015) 040 [arXiv: 1410.2650] [INSPIRE].

[14] Ö. Kelekci, Y. Lozano, N.T. Macpherson and E. Ó. Colgáin, Supersymmetry and non-Abelian T-duality in type-II supergravity, Class. Quant. Grav. 32 (2015) 035014 [arXiv:1409.7406] [INSPIRE].

[15] L.A. Pando Zayas, V.G.J. Rodgers and C.A. Whiting, Supergravity solutions with AdS $S_{4}$ from non-Abelian T-dualities, JHEP 02 (2016) 061 [arXiv:1511.05991] [INSPIRE].

[16] G. Itsios, Y. Lozano, J. Montero and C. Núñez, The AdS $S_{5}$ non-Abelian T-dual of Klebanov-Witten as a $\mathcal{N}=1$ linear quiver from M5-branes, JHEP 09 (2017) 038 [arXiv: 1705.09661] [INSPIRE].

[17] R. Borsato and L. Wulff, Non-abelian T-duality and Yang-Baxter deformations of Green-Schwarz strings, JHEP 08 (2018) 027 [arXiv: 1806.04083] [INSPIRE].

[18] G. Arutyunov, S. Frolov, B. Hoare, R. Roiban and A.A. Tseytlin, Scale invariance of the $\eta$-deformed $A d S_{5} \times S^{5}$ superstring, T-duality and modified type-II equations, Nucl. Phys. B 903 (2016) 262 [arXiv:1511.05795] [INSPIRE].

[19] L. Wulff and A.A. Tseytlin, $\kappa$-symmetry of superstring $\sigma$-model and generalized $10 d$ supergravity equations, JHEP 06 (2016) 174 [arXiv: 1605.04884] [INSPIRE].

[20] M. Gasperini, R. Ricci and G. Veneziano, A Problem with nonAbelian duality?, Phys. Lett. B 319 (1993) 438 [hep-th/9308112] [INSPIRE].

[21] S. Elitzur, A. Giveon, E. Rabinovici, A. Schwimmer and G. Veneziano, Remarks on nonAbelian duality, Nucl. Phys. B 435 (1995) 147 [hep-th/9409011] [InSPIRE].

[22] M. Hong, Y. Kim and E. Ó. Colgáin, On non-Abelian T-duality for non-semisimple groups, Eur. Phys. J. C 78 (2018) 1025 [arXiv:1801.09567] [InSPIRE].

[23] A. Giveon, M. Porrati and E. Rabinovici, Target space duality in string theory, Phys. Rept. 244 (1994) 77 [hep-th/9401139] [INSPIRE].

[24] M. Fukuma, T. Oota and H. Tanaka, Comments on T dualities of Ramond-Ramond potentials on tori, Prog. Theor. Phys. 103 (2000) 425 [hep-th/9907132] [INSPIRE].

[25] Y. Sakatani, Type II DFT solutions from Poisson-Lie T-duality/plurality, arXiv:1903.12175 [INSPIRE].

[26] M. Bugden, Non-abelian T-folds, JHEP 03 (2019) 189 [arXiv:1901.03782] [INSPIRE].

[27] A. Catal-Ozer, Non-Abelian T-duality as an $O(d, d)$ transformation, APCTP, Pohang, Korea, (2016) [https://www.apctp.org/plan.php/duality/1341].

[28] A.A. Tseytlin, Duality symmetric closed string theory and interacting chiral scalars, Nucl. Phys. B 350 (1991) 395 [inSPIRE].

[29] A.A. Tseytlin, Duality Symmetric Formulation of String World Sheet Dynamics, Phys. Lett. B 242 (1990) 163 [INSPIRE].

[30] W. Siegel, Two vierbein formalism for string inspired axionic gravity, Phys. Rev. D 47 (1993) 5453 [hep-th/9302036] [INSPIRE]. 
[31] W. Siegel, Superspace duality in low-energy superstrings, Phys. Rev. D 48 (1993) 2826 [hep-th/9305073] [INSPIRE].

[32] W. Siegel, Manifest duality in low-energy superstrings, in International Conference on Strings 93, Berkeley, California, 24-29 May 1993, pp. 353-363 (1993) [hep-th/9308133] [InSPIRE].

[33] C. Hull and B. Zwiebach, Double Field Theory, JHEP 09 (2009) 099 [arXiv:0904.4664] [INSPIRE].

[34] O. Hohm, C. Hull and B. Zwiebach, Generalized metric formulation of double field theory, JHEP 08 (2010) 008 [arXiv: 1006.4823] [INSPIRE].

[35] B. Zwiebach, Double Field Theory, T-duality and Courant Brackets, Lect. Notes Phys. 851 (2012) 265 [arXiv:1109.1782] [INSPIRE].

[36] D. Geissbuhler, D. Marques, C. Núñez and V. Penas, Exploring Double Field Theory, JHEP 06 (2013) 101 [arXiv: 1304.1472] [INSPIRE].

[37] O. Hohm, D. Lüst and B. Zwiebach, The Spacetime of Double Field Theory: Review, Remarks and Outlook, Fortsch. Phys. 61 (2013) 926 [arXiv:1309.2977] [INSPIRE].

[38] O. Hohm, S.K. Kwak and B. Zwiebach, Double Field Theory of Type II Strings, JHEP 09 (2011) 013 [arXiv: 1107.0008] [INSPIRE].

[39] Y. Sakatani, S. Uehara and K. Yoshida, Generalized gravity from modified DFT, JHEP 04 (2017) 123 [arXiv: 1611.05856] [INSPIRE].

[40] J.-i. Sakamoto, Y. Sakatani and K. Yoshida, Weyl invariance for generalized supergravity backgrounds from the doubled formalism, PTEP 2017 (2017) 053B07 [arXiv:1703.09213] [INSPIRE].

[41] D. Lüst and D. Osten, Generalised fluxes, Yang-Baxter deformations and the $O(d, d)$ structure of non-abelian T-duality, JHEP 05 (2018) 165 [arXiv:1803.03971] [INSPIRE].

[42] S. Demulder, F. Hassler and D.C. Thompson, Doubled aspects of generalised dualities and integrable deformations, JHEP 02 (2019) 189 [arXiv:1810.11446] [INSPIRE].

[43] C. Klimčík and P. Ševera, Dual nonAbelian duality and the Drinfeld double, Phys. Lett. B 351 (1995) 455 [hep-th/9502122] [INSPIRE].

[44] C. Klimčík, Poisson-Lie T duality, Nucl. Phys. Proc. Suppl. 46 (1996) 116 [hep-th/9509095] [INSPIRE].

[45] F. Hassler, Poisson-Lie T-duality in Double Field Theory, arXiv:1707.08624 [InSPIRE].

[46] R. Blumenhagen, F. Hassler and D. Lüst, Double Field Theory on Group Manifolds, JHEP 02 (2015) 001 [arXiv: 1410.6374] [INSPIRE].

[47] R. Blumenhagen, P. du Bosque, F. Hassler and D. Lüst, Generalized Metric Formulation of Double Field Theory on Group Manifolds, JHEP 08 (2015) 056 [arXiv:1502.02428] [INSPIRE].

[48] F. Hassler, The Topology of Double Field Theory, JHEP 04 (2018) 128 [arXiv:1611.07978] [INSPIRE].

[49] J. Scherk and J.H. Schwarz, How to Get Masses from Extra Dimensions, Nucl. Phys. B 153 (1979) 61 [INSPIRE].

[50] J. Scherk and J.H. Schwarz, Spontaneous Breaking of Supersymmetry Through Dimensional Reduction, Phys. Lett. 82B (1979) 60 [InSPIRE].

[51] D. Geissbuhler, Double Field Theory and $N=4$ Gauged Supergravity, JHEP 11 (2011) 116 [arXiv: 1109.4280] [INSPIRE]. 
[52] G. Aldazabal, W. Baron, D. Marques and C. Núñez, The effective action of Double Field Theory, JHEP 11 (2011) 052 [Erratum ibid. 11 (2011) 109] [arXiv:1109.0290] [INSPIRE].

[53] M. Graña and D. Marques, Gauged Double Field Theory, JHEP 04 (2012) 020 [arXiv: 1201.2924] [INSPIRE].

[54] A. Catal-Ozer, Duality Twisted Reductions of Double Field Theory of Type II Strings, JHEP 09 (2017) 044 [arXiv: 1705.08181] [INSPIRE].

[55] A. Catal-Ozer, Lunin-Maldacena deformations with three parameters, JHEP 02 (2006) 026 [hep-th/0512290] [INSPIRE].

[56] E. Bergshoeff, R. Kallosh, T. Ortín, D. Roest and A. Van Proeyen, New formulations of $D=10$ supersymmetry and D8-O8 domain walls, Class. Quant. Grav. 18 (2001) 3359 [hep-th/0103233] [INSPIRE].

[57] S. Mukai, Symplectic Structure of the Moduli Space of Sheaves on an Abelian or K3 Surface, Invent. Math. 77 (1984) 101.

[58] A. Catal-Ozer, Massive deformations of Type IIA theory within double field theory, JHEP 02 (2018) 179 [arXiv:1706.08883] [INSPIRE].

[59] I. Kawaguchi, T. Matsumoto and K. Yoshida, Jordanian deformations of the $A d S_{5} \times S^{5}$ superstring, JHEP 04 (2014) 153 [arXiv:1401.4855] [INSPIRE].

[60] B. Hoare and A.A. Tseytlin, Homogeneous Yang-Baxter deformations as non-abelian duals of the $A d S_{5}$ G-model, J. Phys. A 49 (2016) 494001 [arXiv:1609.02550] [INSPIRE].

[61] R. Borsato and L. Wulff, Integrable Deformations of T-Dual $\sigma$ Models, Phys. Rev. Lett. 117 (2016) 251602 [arXiv: 1609.09834] [INSPIRE].

[62] J.-i. Sakamoto, Y. Sakatani and K. Yoshida, Homogeneous Yang-Baxter deformations as generalized diffeomorphisms, J. Phys. A 50 (2017) 415401 [arXiv:1705.07116] [INSPIRE].

[63] I. Bakhmatov, E. Ó Colgáin, M.M. Sheikh-Jabbari and H. Yavartanoo, Yang-Baxter Deformations Beyond Coset Spaces (a slick way to do TsT), JHEP 06 (2018) 161 [arXiv: 1803.07498] [INSPIRE].

[64] J.-I. Sakamoto and Y. Sakatani, Local $\beta$-deformations and Yang-Baxter $\sigma$-model, JHEP 06 (2018) 147 [arXiv : 1803.05903] [InSPIRE].

[65] I. Bakhmatov and E.T. Musaev, Classical Yang-Baxter equation from $\beta$-supergravity, JHEP 01 (2019) 140 [arXiv:1811.09056] [INSPIRE].

[66] T. Araujo, I. Bakhmatov, E. Ó. Colgáin, J. Sakamoto, M.M. Sheikh-Jabbari and K. Yoshida, Yang-Baxter $\sigma$-models, conformal twists and noncommutative Yang-Mills theory, Phys. Rev. D 95 (2017) 105006 [arXiv:1702.02861] [INSPIRE].

[67] T. Araujo, I. Bakhmatov, E. Ó. Colgáin, J.-i. Sakamoto, M.M. Sheikh-Jabbari and K. Yoshida, Conformal twists, Yang-Baxter $\sigma$-models $\& 3$ holographic noncommutativity, J. Phys. A 51 (2018) 235401 [arXiv:1705.02063] [InSPIRE].

[68] A. Çatal Özer and S. Tunal, Yang-Baxter Deformation as an $O(d, d)$ Transformation, arXiv: 1906.09053 [INSPIRE]. 